\title{
Benchmarking ICRF full-wave solvers for ITER
}

\author{
R.V. Budny ${ }^{1}$, L. Berry ${ }^{2}$, R. Bilato ${ }^{3}$, P. Bonoli ${ }^{4}$, M. Brambilla ${ }^{3}$, \\ R.J. Dumont ${ }^{5}$, A. Fukuyama ${ }^{6}$, R. Harvey ${ }^{7}$, E.F. Jaeger ${ }^{8}$, \\ K. Indireshkumar ${ }^{1}$, E. Lerche ${ }^{9}$, D. McCune ${ }^{1}$, C.K. Phillips ${ }^{1}$, \\ V. Vdovin ${ }^{10}$, J. Wright ${ }^{4}$ and members of the ITPA-IOS \\ ${ }^{1}$ PPPL, PO Box 451, Princeton, NJ 08543, USA \\ 2 ORNL, PO Box 2008, Oak Ridge, TN 37831, USA \\ ${ }^{3}$ Max-Planck-Institut für Plasmaphysik, Garching, Germany \\ ${ }^{4}$ MIT Plasma Science and Fusion Center, 77 Mass. Avenue, Cambridge, MA 02139, USA \\ ${ }^{5}$ CEA, IRFM, F-13108 Saint-Paul-lez-Durance, France \\ ${ }^{6}$ Department of Nuclear Engineering, Kyoto University, Kyoto, 606-8501, Japan \\ ${ }^{7}$ CompX, Box 2672, Del Mar, CA 92014, USA \\ ${ }^{8}$ XCEL Engineering Inc., 1066 Commerce Park Dr., Oak Ridge, TN 37830, USA \\ ${ }^{9}$ LPP-ERM/KMS, Association Euratom-Belgian State, TEC Partner, Brussels, Belgium \\ ${ }^{10}$ RRC Kurchatov Institute Tokamaks Physics Institute, Russia \\ E-mail: budny@princeton.edu
}

Received 17 January 2011, accepted for publication 6 January 2012

Published 1 February 2012

Online at stacks.iop.org/NF/52/023023

\begin{abstract}
Benchmarking full-wave solvers for ion-cyclotron range of frequency (ICRF) simulations is performed using plasma profiles and equilibria obtained from integrated self-consistent modelling predictions of four ITER plasmas. One is for a high-performance baseline (5.3 T, 15 MA) DT H-mode. The others are for half-field, half-current plasmas of interest for the pre-activation phase with bulk plasma ion species being either hydrogen or $\mathrm{He}^{4}$. The predicted profiles are used by six full-wave solver groups to simulate the ICRF electromagnetic fields and heating, and by three of these groups to simulate the current drive. Approximate agreement is achieved by four of the solvers for the heating power partitions for the DT and $\mathrm{He}^{4}$ cases. Factor of two or more disagreements are found for the heating power partitions for the cases with second harmonic $\mathrm{He}^{3}$ heating in bulk $\mathrm{H}$ cases. Approximate agreement is achieved simulating the ICRF current-drive 1D profiles.
\end{abstract}

\section{Introduction}

Ion-cyclotron range of frequency (ICRF) heating is planned to be an important component of the ITER heating system. The planned heating power is up to $20 \mathrm{MW}$ and the range of frequency is $40-55 \mathrm{MHz}$. Simulations of ICRF heating, current-drive and torque profiles are needed for estimating the effectiveness of the ICRF system in helping to create and sustain high fusion power. To get realistic plasmas for ICRF simulations and for performance predictions, integrated modelling is needed since the plasma profiles and applied heating and current drive are strongly coupled. Benchmarking codes used for simulating heating and current drive is important for verifying and assessing confidence in the simulations and in the predictions.

Time-dependent integrated modelling is important for ITER design and for planning experiments. Some of the ITER predictive modelling is done employing ad hoc profiles for the ICRF, or simple, unbenchmarked ICRF models. Credible and useful modelling needs to balance physics fidelity and numerical resolution with run speeds. Simulating some ICRF effects such as mode conversion (MC), and some plasma regimes require much greater spatial resolution than others. Hence an important byproduct of benchmarking is an indication of the numerical resolution needed for accurate fullwave simulations, and also of the level of model sophistication needed to capture the important physics.

The purpose of this study is to compare the predictions of a group of ICRF full-wave solvers using four ITER plasma scenarios obtained from realistic integrated modelling predictions. The goals of these comparisons are to indicate (1) which full-wave solvers and numerical resolutions are credible for use in predictive codes, (2) ITER plasma regimes requiring special care and (3) where solvers need improvements.

Several phases of plasma operation planned for ITER are discussed in [1]. A pre-activation phase is desired for 
Table 1. Summary of the benchmarking cases predicted by PTRANSP.

\begin{tabular}{lllll}
\hline Case & $\begin{array}{l}\text { Case 1 } \\
(\mathrm{DT})\end{array}$ & $\begin{array}{l}\text { Case 2 } \\
(\mathrm{H})\end{array}$ & $\begin{array}{l}\text { Case 3 } \\
(\mathrm{H})\end{array}$ & $\begin{array}{l}\text { Case 4 } \\
\left(\mathrm{He}^{4}\right)\end{array}$ \\
\hline Bulk ion species & $\mathrm{DT}$ & $\mathrm{H}$ & $\mathrm{H}$ & $\mathrm{He}^{4}$ \\
Impurity species & $\begin{array}{l}\text { ash, Ar, Be } \\
\text { D-beam, } \\
\text { Fast ion species }\end{array}$ & $\begin{array}{l}\mathrm{C} \text {-beam } \\
\text { alphas }\end{array}$ & $\begin{array}{l}\mathrm{C} \\
\mathrm{H} \text {-beam }\end{array}$ & $\begin{array}{l}\mathrm{C} \\
\text { none }\end{array}$ \\
$B_{\mathrm{T}}(\mathrm{T})$ & 5.314 & 2.678 & 2.665 & 2.665 \\
$I_{\mathrm{p}}(\mathrm{MA})$ & 15.0 & 7.5 & 7.5 & 7.5 \\
$n_{\mathrm{e}}(0)\left(10^{20} \mathrm{~m}^{-3}\right)$ & 1.05 & 0.46 & 0.46 & 0.46 \\
$T_{\mathrm{i}}(0)(\mathrm{keV})$ & 27.5 & 10 & 12 & 13.5 \\
$T_{\mathrm{e}}(0)(\mathrm{keV})$ & 25 & 14 & 15 & 12.5 \\
$T_{\text {ped }}(\mathrm{keV})$ & 5.3 & 1.5 & 2.5 & 1.8 \\
$\beta_{\mathrm{n}}$ & 2.0 & 1.5 & 1.8 & 1.2 \\
$P_{\mathrm{NNBI}}(\mathrm{MW})$ & 17.0 & 17.0 & 33.0 & 0.0 \\
$P_{\mathrm{EC}}(\mathrm{MW})$ & 20.0 & 20.0 & 20.0 & 20.0 \\
$P_{\mathrm{IC}}(\mathrm{MW})$ & 10.0 & 20.0 & 20.0 & 20.0 \\
ICRF frequency & 52.5 & 52.5 & 52.5 & 42.0 \\
$(\mathrm{MHz})$ & & & & \\
Minority species & $\mathrm{He}$ & $\mathrm{He}$ & $\mathrm{He}^{3}$ & $\mathrm{H}$ \\
$n_{\text {minor }} / n_{\mathrm{e}}$ & 0.02 & 0.03 & 0.20 & 0.20 \\
$E_{\|}($minor) $(\mathrm{MJ})$ & 1.6 & 0.4 & 3.7 & 3.7 \\
$E_{\perp}(\mathrm{minor})(\mathrm{MJ})$ & 3.4 & 1.5 & 10.0 & 9.5 \\
\hline
\end{tabular}

checking, testing and calibrating the heating, diagnostics, stability, control, fuelling, exhaust and safety systems. It will be especially helpful if the H-mode can be obtained in this phase for studying first wall heating, particle retention, ELM effects, disruptions and plasma control. There are indications that the H-mode might be achieved in hydrogen or $\mathrm{He}^{4}$ dominated plasmas with low field and density, and with the planned auxiliary heating power.

The auxiliary heating and current-drive systems being designed for both the pre-activation and initial DT phases are negative-ion neutral beam injection (NNBI), ICRF and electron-cyclotron range of frequency (ECRF). NNBI simulation codes have been extensively benchmarked for present experiments and for ITER [2,3]. Likewise ECRF simulations have been benchmarked in present experiments and in ITER [4]. Two of the ICRF solvers have been benchmarked for an ITER plasma [15], and the results from that study are similar to the results for case 1 below.

In recent years the state of the art has developed considerably in terms of the level of physics sophistication available in ICRF full-wave solvers. For example capabilities now exist to treat MC to short wavelength modes, to include a realistic scrape-off layer, and to employ numerical fast particle distributions. Advances in parallel processing have allowed solutions with high resolution. This paper employs these collective advanced simulation capabilities to assess several important ICRF-heating scenarios in ITER.

\section{Benchmark cases}

The PTRANSP code [5-8] is used to generate predictions of ITER plasmas for inputs in the benchmarking cases. The cases are listed in table 1. The PTRANSP predictions are time-dependent, integrated and self-consistent in that the heating, current drive and beam torques are calculated using predicted plasma profiles. Fast ions, multiple impurities, and

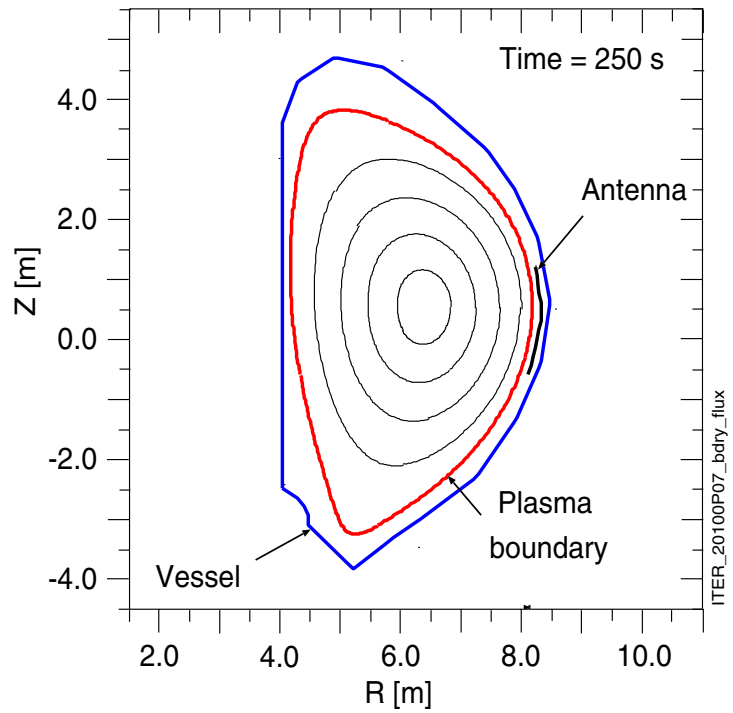

Figure 1. Assumed boundary and computed flux surfaces for the DT case 1 .

the up/down asymmetric geometry of the flux surfaces are included. An example of the geometry anticipated in ITER is shown in figure 1 .

The cases include a high-performance baseline $\left(B_{\mathrm{TF}}=\right.$ 5.3 T, $I_{\mathrm{p}}=15 \mathrm{MA}$ ) DT plasma (case 1) and plasmas for the pre-activation phase with half-field and half-current and either bulk $\mathrm{H}$ (cases 2 and 3 ) or $\mathrm{He}^{4}$ (case 4). The DT case 1 is taken from [7]. For case 1 the dominant ICRF absorption mechanisms are fundamental $\mathrm{He}^{3}$ ion-cyclotron absorption and electron Landau damping (ELD). Case 2 is predicted to be L-mode and the others are predicted to be H-mode. For the pre-activation bulk $\mathrm{H}$ cases the dominant absorption of ICRF power is via ELD, second harmonic heating of the $\mathrm{He}^{3}$ minority with frequency twice the ion-cyclotron frequency $\left(\omega=2 \Omega_{\mathrm{c}}\right)$, and first-harmonic heating of the majority $\mathrm{H}\left(\omega=\Omega_{\mathrm{c}}\right)$.

The second harmonic heating fraction is calculated to increase by increasing either the density or the energy of the $\mathrm{He}^{3}$. Since case 2 is predicted to be in L-mode, the NNBI power is raised from 17 to $33 \mathrm{MW}$ for case 3 to attempt to achieve $\mathrm{H}$ mode. Also the fraction of $\mathrm{He}^{3}$ is raised from $n_{\min } / n_{\mathrm{e}}$ of 0.03 to 0.20 . This case is predicted to be marginally in $\mathrm{H}$-mode. Both increases result in increased $\mathrm{He}^{3}$ heating.

Cases 2 and 3 (accelerating $\mathrm{He}^{3}$ at its second harmonic) are numerical explorations of a heating scenario under assessment but currently not considered a main scenario for ITER. Experiments with this scheme using low concentrations of $\mathrm{He}^{3}$ have been conducted in JET [9] and Tore Supra [10]. The results have not been encouraging. In JET either low electron density (conducive to tail formation) or high $\mathrm{He}^{3}$ concentrations $(\geqslant 15 \%)$ are needed in order to see increases in the ion temperature. Perhaps the heating power in these experiments was not sufficiently high to achieve high $\mathrm{He}^{3}$ energies. These cases are included in the benchmarking since they are especially challenging.

Another pre-activation scenario is case 4 with $\mathrm{H}$ minority at half-field in a bulk $\mathrm{He}^{4}$ plasma with fundamental absorption at $42 \mathrm{MHz}$. One rationale for this case is that it could be important for obtaining $\mathrm{H}$-mode in the pre-activation phase, 

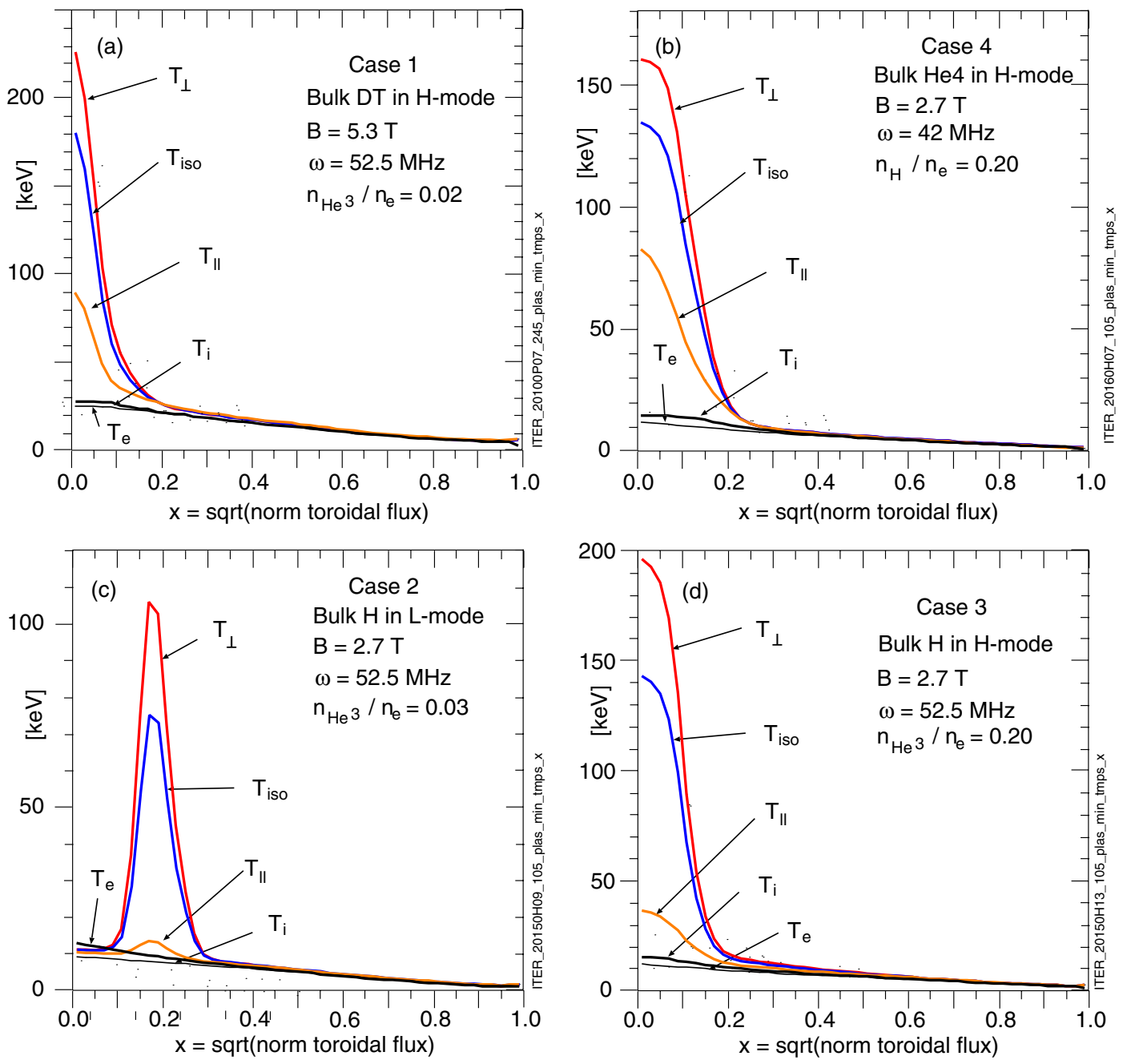

Figure 2. Temperature profiles for the benchmark cases, computed from PTRANSP using GLF23 for the plasma and FFPRF for the minority ions using equations (1) and (2).

especially if the $\mathrm{H}$-mode threshold favours bulk $\mathrm{He}^{4}$ over bulk hydrogen. Scalings for the $\mathrm{L} \rightarrow \mathrm{H}$ power threshold $P_{\mathrm{LH}}$ in $\mathrm{He}^{4}$ plasmas are controversial, but some tokamaks report lower $\mathrm{H}$-mode threshold in $\mathrm{He}^{4}$ than in $\mathrm{H}$ [11]. Cases 1, 3 and 4 achieve $\mathrm{H}$-mode with extrapolations of ITPA database results for $P_{\mathrm{LH}}$ [12]. Also, case 4 appears to be relatively easy for benchmarking since indications are that case 4 will have strong single-pass absorption at the fundamental $\mathrm{H}$ cyclotron resonance and will not have strong $\mathrm{MC}$ (which is not treated in some of the codes involved in the benchmarking).

The PTRANSP outputs, used as inputs for the fullwave solvers, are the plasma equilibria and profiles of the densities and temperatures of the thermal plasma (including impurities) and fast-ion species. The predicted plasma profiles are provided for the full-wave codes in the format of 'plasma state' netcdf files [13]. Alternatively the equilibria inputs are provided as 'g-eqdisk' files [14], and as a set of ASCII files with the equilibria in the form of Fourier poloidal moments specifying the $(R, Z)$ values of constant magnetic flux surfaces.

Perpendicular and parallel energy density profiles $e_{\perp}$ and $e_{\|}$of the minority, beam, and fast alpha ions are also provided. The total perpendicular and parallel energies of the minority ions are given in table 1 . Their effective temperatures are defined using either an isotropic profile

$$
T_{\text {iso }}=2 / 3 \cdot\left(e_{\perp}+e_{\|}\right) / n_{\text {fast }},
$$

or two anisotropic profiles:

$$
T_{\perp}=e_{\perp} / n_{\text {fast }}, \quad T_{\|}=2 \cdot e_{\|} / n_{\text {fast }},
$$

where $n_{\text {fast }}$ is the fast-ion density. Profiles of the thermal ion and electron temperatures $T_{\mathrm{i}}$ and $T_{\mathrm{e}}$ and of $T_{\perp}$ and $T_{\|}$for the minority ions are shown in figure 2 . Note that relatively large 'tail' temperatures and large differences between $T_{\perp}, T_{\|}$and $T_{\text {iso }}$ are predicted.

The benchmark cases assume a simple toroidal spectrum for the ICRF at the antenna. For most of the results two wavenumbers are used with $n_{\phi}= \pm 27$, (symmetric in $\phi$ ). For current-drive simulations only one peak with $n_{\phi}=+27$ is assumed. The spectral components used in each code to represent the electric field correspond to a unique $k_{\phi}=n_{\phi} / R$ which is $4.229 \mathrm{~m}^{-1}$ at the major radius of the magnetic axis for case 1. The equivalent parallel index is 3.843. Different approximations for the antenna, scrape-off layer and vacuum vessel are assumed by the different wave-solvers. More details about the assumptions used in PTRANSP are given in appendix A. 
Table 2. Summary of full-wave solvers and their ability to treat general geometry, MC, order of FLR approximations, and numerical methods.

\begin{tabular}{|c|c|c|c|c|}
\hline Code & $\begin{array}{l}\text { General } \\
\text { geom? }\end{array}$ & MC? & FLR & Numerical methods \\
\hline AORSA & Yes & Yes & all orders & $\begin{array}{l}\text { Fourier collocation } \\
\text { in } k_{x}, k_{y}, k_{\phi}\end{array}$ \\
\hline EVE & Yes & Yes & 2 nd order & $\begin{array}{l}\text { Variation method; tor } \\
\text { and pol modes; } \\
\text { radial finite elements }\end{array}$ \\
\hline CYRANO & No & No & 2 nd order & $\begin{array}{l}\text { Variation method; } \\
\text { tor and pol modes; } \\
\text { radial finite elements }\end{array}$ \\
\hline PSTELION & Approx & Yes & 2 nd order & $\begin{array}{l}\text { Finite differences in } \\
\text { radial coordinate }\end{array}$ \\
\hline TORIC & Yes & Yes & 2 nd order & $\begin{array}{l}\text { Variation method; } \\
\text { tor and pol modes; } \\
\text { radial finite elements }\end{array}$ \\
\hline TASK/WM & No & No & 2nd order & $\begin{array}{l}\text { Tor and pol modes; } \\
\text { radial finite element }\end{array}$ \\
\hline
\end{tabular}

\section{Full-wave solvers}

The profiles and equilibria predictions are used independently by six groups to simulate the ICRF electromagnetic fields and plasma heating. The codes include AORSA $[15,16]$, CYRANO [17, 18], EVE [19], PSTELION [20], TASK/WM [21] and TORIC (version 6) [22, 23], (version 5 is used for the PTRANSP-generated inputs for the simulations). A summary of approximations and numerical methods is given in table 2 , and more details are given in appendix B.

Referring to table 2, all but one of the solvers use a finite-Larmor radius (FLR) approximation for the conductivity operator. This approximation assumes that the ion gyro-radius is small relative to the perpendicular wavelength, i.e. that $k_{\perp} \rho_{\mathrm{i}}<<1$ with $k_{\perp}$ the wavenumber perpendicular to $B$, and $\rho_{\mathrm{i}}$ the ion radius. AORSA uses an integral conductivity formulation valid at all ion-cyclotron harmonics and arbitrary perpendicular wavelengths. Thus AORSA can calculate MC to ion Bernstein waves (IBWs) and ion-cyclotron waves (ICWs) rigorously. The EVE, TORIC and PSTELION solvers employ a reduced description of MC, while the CYRANO and TASK/WM codes do not treat MC. All of the full-wave solvers in this study include variations (upshift and downshift) of the parallel wavenumber. The ELD includes both parallel Landau absorption, electron transit time magnetic pumping TTMP, as well as the effect of a cross-term between the ELD and TTMP caused by field compression.

Numerical methods utilized in these codes vary with AORSA being completely spectral, e.g. $k_{x}, k_{y}, k_{\phi}$. The solvers EVE, CYRANO, TASK/WM and TORIC are semi-spectral, employing finite elements for the radial dimension and Fourier representations in the poloidal and toroidal directions. EVE uses different dependent variables. PSTELION is also semispectral, but employs finite differences in the radial direction. The AORSA, EVE and TORIC solvers use general geometry equilibrium representations whereas CYRANO, PSTELION and TASK/WM use simpler equilibrium representations. There are different choices for which distribution functions can be used to calculate the conductivity operator in the wavesolver: mono-Maxwellian (equation (1)), bi-Maxwellians (equation (2)), or a numerical phase-space distribution.
Table 3. PTRANSP-FPPRF results for heating powers of the ICRF, and for the minority species heating to the thermal plasma. The sums of the last two rows are approximately the total ICRF-minority heating listed one line above.

\begin{tabular}{lllll}
\hline Bulk ion species & DT case 1 & $\mathrm{H}$ case 2 & $\mathrm{H}$ case 3 & $\mathrm{He}^{4}$ case 4 \\
\hline $\begin{array}{l}\text { ICRF } \\
(\mathrm{MW})\end{array}$ & 10.0 & 20.0 & 20.0 & 20.0 \\
$\begin{array}{l}\text { ICRF-electrons } \\
(\mathrm{MW})\end{array}$ & 3.7 & 11.1 & 3.9 & 4.0 \\
$\begin{array}{l}\text { ICRF-thermal } \\
\text { ions (MW) }\end{array}$ & 1.4 & 4.9 & 1.0 & 0.2 \\
$\begin{array}{l}\text { ICRF-minority } \\
(\mathrm{MW})\end{array}$ & 5.3 & 4.1 & 15.4 & 16.1 \\
$\begin{array}{l}\text { Minority-electrons } \\
(\mathrm{MW})\end{array}$ & 1.2 & 1.8 & 10.5 & 10.2 \\
$\begin{array}{c}\text { Minority-thermal } \\
\text { ions (MW) }\end{array}$ & 4.0 & 2.3 & 5.2 & 5.5 \\
\hline
\end{tabular}

Parameters needed for quantitative comparisons of the benchmarking results include the locations of resonance layers, zero-dimensional results such as the heating partitions, one-dimensional results such as heating profiles and electromagnetic fields along chords. Two-dimensional contours of heating and electromagnetic fields are very useful for giving insight about the solutions, and for checking that the geometry is being read in correctly by the full-wave solvers. It is important to compare the locations of resonance layers. Computing which locations are inside the plasma is complicated by the general shape of the boundary, and by the fact that in time-evolving simulations the boundary shifts. Thus simple algorithms that check only along the midplane are insufficient.

Part of the research for this benchmarking involved refining and standardizing methods for inputting data to the full-wave solvers. For instance, one improvement to some of the codes is the ability to read in numerical equilibria and plasma profiles. In addition to being important for the benchmarking comparisons, the ability to read numerical equilibria and fits to data are important for testing the solvers with experimental data.

\section{Fokker-Planck solvers}

The minority ion phase-space distributions and heating need to be predicted for accurate simulations. This is especially complicated in scenarios where other fast ions (beam ions and fusion alphas) are resonant with the ICRF. In such cases, and also if FLR effects are important, Monte Carlo techniques may be needed for accurate coupling of the wave heating. Since Monte Carlo techniques are very challenging, especially in the presence of multiple fast-ion species, Fokker-Planck techniques are typically used. Various Fokker-Planck codes have been coupled to full-wave solvers. These can have complications of averaging over banana orbits and loss of FLR effects.

The Fokker-Planck module in PTRANSP (FPPRF) [24] uses up/down asymmetric equilibria, and computes the minority ion phase-space distribution. In FPPRF, the bounceaveraged Fokker-Planck code FPP is coupled to the TORIC solver as follows. The electric wave fields from TORIC are used to construct an approximate quasi-linear ICRF diffusion 
Table 4. Major radii of the magnetic axes for the four cases and the locations of ion resonances measured from the magnetic axis. Ions with the same $A / Z$ ratio have the same resonance locations so, for instance, D-beam ions, ash, fusion alphas, and any impurity with ratio 2 have the same location as that of thermal D.

\begin{tabular}{lll}
\hline Ion species & Fund resonance & 2nd harmonic resonance \\
\hline Case 1 bulk DT & Axis at $6.385 \mathrm{~m}$ & \\
$A / Z=2 / 1$ & $-1.488 \mathrm{~m}$ & $3.437 \mathrm{~m}$ (outside boundary) \\
$A / Z=3 / 1$ & $-3.111 \mathrm{~m}$ (outside boundary) & $0.018 \mathrm{~m}$ \\
$A / Z=3 / 2$ & $0.018 \mathrm{~m}$ & $6.711 \mathrm{~m}$ (outside boundary) \\
Case 2 bulk H & Axis at $6.320 \mathrm{~m}$ & \\
$A / Z=1 / 1$ & $-1.368 \mathrm{~m}$ & $3.721 \mathrm{~m}$ (outside boundary) \\
$A / Z=2 / 1$ & $-3.810 \mathrm{~m}$ (outside boundary) & $-1.368 \mathrm{~m}$ \\
$A / Z=3 / 2$ & $-2.973 \mathrm{~m}$ (outside boundary) & $0.364 \mathrm{~m}$ \\
Case 3 bulk H & Axis at $6.475 \mathrm{~m}$ & \\
$A / Z=1 / 1$ & $-1.590 \mathrm{~m}$ & $3.033 \mathrm{~m}$ (outside boundary) \\
$A / Z=2 / 1$ & $-4.098 \mathrm{~m}$ (outside boundary) & $-1.590 \mathrm{~m}$ \\
$A / Z=3 / 2$ & $-3.306 \mathrm{~m}$ (outside boundary) & $-0.096 \mathrm{~m}$ \\
Case 4 bulk He 4 & Axis at $6.419 \mathrm{~m}$ & \\
$A / Z=2 / 1$ & $-3.401 \mathrm{~m}$ (outside boundary) & $-0.281 \mathrm{~m}$ \\
$A / Z=1 / 1$ & $-0.281 \mathrm{~m}$ & $5.653 \mathrm{~m}$ (outside boundary) \\
\hline
\end{tabular}

Table 5. Locations of the ion-ion resonance layers and the ion-ion cutoff.

\begin{tabular}{lll}
\hline Ion species & Ion-ion resonance & Ion-ion cutoff \\
\hline Case 1 & $-0.064 \mathrm{~m}$ & $-0.084 \mathrm{~m}$ \\
Case 4 & $-1.076 \mathrm{~m}$ & $-0.859 \mathrm{~m}$ \\
\hline
\end{tabular}

coefficient following the Kennel-Engelmann [25] formulation. The diffusion coefficient is used in FPP. The energetic ion distribution function from FPP is used to compute an effective tail temperature that is then used as a bi-Maxwellian distribution in the TORIC solvers. The PTRANSP output summarizes this as energy densities $e_{\perp}$ and $e_{\|}$used for the biMaxwellian assumption equation (2). Results for the predicted minority temperatures are shown in figure 2 , and results for the ICRF and minority heating of the thermal plasma are given in table 3.

The CQL3D Fokker-Planck solver [26] which can be run with AORSA can compute the phase-space distribution in energy and pitch angle using up/down symmetric equilibria. Unlike TORIC the numerical distribution function evolved by CQL3D is used directly in AORSA to re-evaluate the plasma response. In turn the wave fields in AORSA are used to evaluate the RF diffusion coefficient. Although benchmarking of the Fokker-Planck modules is not part of this paper, AORSACQL3D simulation results are included for comparisons with PTRANSP-FPPRF.

\section{Benchmark results}

The locations of the resonance surfaces can depend sensitively on the ICRF frequency. The locations computed by PTRANSP are given in table 4 . The locations of the ion-ion resonances and ion-ion cutoff for cases 1 and 4 are given in table 5 . These locations agree approximately with those found by the fullwave solvers.

The heating power partitions among the plasma species depend sensitively on details such as the density and effective temperature of the minority ions. Results for the direct ICRF heating from the full-wave solvers for case 1 are shown in table 6. The first row gives results from the PTRANSPTORIC(v5) predictions used as inputs for the other full-wave solvers. These partitions differ from those of the other fullwave solvers given below, including those of TORIC(v6). The newer version has an improved numerical treatment of the magnetic axis and vacuum, and in the Fokker-Planck solver and its coupling with TORIC. The main cause of differences between the PTRANSP-TORIC(v5) and standalone TORIC(v6) results is the low poloidal resolution in PTRANSP (only 31 modes) and in the use of a bi-Maxwellian distribution in PTRANSP versus the isotropic distribution in TORIC(v6).

Comparing the benchmarking for case 1 , the heating partitions for the minority $\mathrm{He}^{3}$ and electron absorption are in approximate agreement, except for the PSTELION results with considerably lower electron heating and higher minority ion heating, and to a much lesser extent the CYRANO results with higher electron heating and lower minority ion heating. Despite the fact that there are significant variations in the absorption on the thermal $\mathrm{D}, \mathrm{He}^{4}$ ash, and impurity ions among the codes, these differences are not thought to be important since the absolute level of absorption in those cases is typically less than $1 \%$. Results for the heating partitions for the preactivation case 4 in bulk $\mathrm{He}^{4}$ plasma are also in approximate agreement, as shown in table 7. Again, the total ICRF-ion heating dominates.

The reason for the significantly lower electron absorption predicted by PSTELION is not understood at this point. This code includes MC as do AORSA, EVE and TORIC, which necessitates a high degree of numerical resolution even in the case of low minority fractions (such as case 1) and certainly in cases 3 and 4 with high minority fraction. Thus it appears that the PSTELION simulation requires higher resolution for accuracy.

Plots of the real and imaginary parts of one of the components of the oscillating electric field $\left(E_{-}\right)$along the major radius are shown in figure 3. Two cases are compared: case 1 with $10 \mathrm{MW}$ ICRF and case 2 with $20 \mathrm{MW}$ at the benchmark time. The ICRF oscillating E and B fields scale 
Table 6. Comparisons of heating partitions (\%) for the case 1 (DT) with $n_{\mathrm{He}^{3}} / n_{\mathrm{e}}=0.02$. CYRANO and PSTELION results are for a simpler, but similar equilibrium. The PTRANSP results are from the runs generating the profile inputs. The TASK/WM results are for a similar equilibrium and profiles. The PTRANSP-aniso, PSTELION, and TORIC-iso partitions to electrons are split to FW and IBWs. The AORSA-CQL3D results are for comparison with PTRANSP-aniso and the benchmarking results.

\begin{tabular}{llllllllll}
\hline Solver & $T$ & Thermal D & Minority & $\mathrm{He}^{4}$ ash & Electrons & $\mathrm{Ar}$ & $\mathrm{Be}$ & D-beams & Fast alphas \\
\hline PTRANSP-aniso & 12.4 & 0.8 & 49.7 & 0.11 & $36.5+0.3$ & 0.1 & 0.1 & 0.02 & 0.12 \\
AORSA-iso & 14.1 & 0.6 & 55.6 & 0.3 & 29.6 & 0.2 & 0.3 & 0.0 & 0.0 \\
CYRANO & 18.0 & 1.0 & 41.0 & $\mathrm{NA}$ & 39.0 & - & - & - & 1.0 \\
EVE-aniso & 12.5 & 0.4 & 48.8 & 0.1 & 36.8 & 1.1 & 0.2 & 0.0 & 0.1 \\
EVE-iso & 12.4 & 0.4 & 48.6 & 0.1 & 37.0 & 1.2 & 0.2 & 0.0 & 0.1 \\
PSTELION & 18.4 & 0.1 & 67.0 & 0.02 & $13.6+0.6$ & - & - & - & - \\
TASK/WM & 15.2 & 1.1 & 50.0 & 0.03 & 25.7 & - & - & - & - \\
TORIC-iso & 16.0 & 0.5 & 51.2 & 0.03 & $31.7+0.7$ & - & - & - & - \\
AORSA-CQL3D & 13.4 & 0.6 & 56.7 & 0.3 & 29.3 & 0.2 & 0.3 & 0.0 & 0.0 \\
\hline
\end{tabular}

Table 7. Comparisons of heating partitions (\%) for pre-activation bulk $\mathrm{He}^{4}$ case 4 with $n_{\mathrm{H}} / n_{\mathrm{e}}=0.20$. No beams are active in this case.

\begin{tabular}{lllll}
\hline Code & $\mathrm{H}$ & Electrons & $\begin{array}{l}\text { Thermal } \\
\mathrm{He}^{4}\end{array}$ & $\begin{array}{l}\text { Carbon } \\
\text { impurity }\end{array}$ \\
\hline PTRANSP-aniso & 78.5 & $20.02+0.02$ & 1.3 & 0.1 \\
AORSA-iso & 81.6 & 17.6 & 0.7 & 0.07 \\
CYRANO-iso & 75 & 25 & 2.0 & $<0.1$ \\
EVE-aniso & 73.7 & 25.2 & 1.0 & 0.1 \\
EVE-iso & 77.5 & 21.1 & 1.3 & 0.1 \\
TORIC-iso & 78.3 & $20.3+0.00$ & 1.4 & - \\
AORSA-CQL3D & 74.3 & 24.0 & 1.5 & 0.2 \\
\hline
\end{tabular}

with the square root of the power, so the magnitudes of the fields are higher for case 2 . The results for case 1 indicate strong single-pass absorption since the magnitudes of the simulated fields are relatively small inboard of the minority resonance near the magnetic axis (table 4). Similar results are seen for case 4 (the other case with firstharmonic minority heating). MC to propagating IBWs appears to be negligible for the DT case 1 , as indicated by the absence of rapid radial oscillations in plots of the real and imaginary parts of $E_{-}(R)$ figure $3(a)$. This reduces the need for high numerical resolution in this case. Simulations with the resolution increased beyond certain values produce nearly identical results, indicating the minimal resolution for accuracy. Resolution is discussed further in section 7.

In contrast to case 1, weak-single-pass absorption is found for cases 2 and 3 with second harmonic minority $\mathrm{He}^{3}$ heating. Plots for the real and imaginary parts of $E_{-}(R)$ for case 2, shown in figures $3(b),(d)$ and $(f)$, have large values on the high-field side beyond the resonance layer. This makes the numerical solutions much more difficult to compare since the single-pass damping at the second harmonic $\mathrm{He}^{3}$ resonance is relatively weak. Consequently ICRF wavefronts undergo multiple passes before complete absorption takes place and the solutions are sensitive to differences in the edge treatments in the various codes. Heating partitions for cases 2 and 3 are given in tables 8 and table 9 . There is a wide variation for the thermal $\mathrm{H}$ partitions in cases 2 and 3.

Comparison of the different codes is further complicated by the fact that only some of the codes include MC. Even in cases where $\mathrm{MC}$ is weak, the presence of mode converted wave fields can affect the wave polarization, especially in cases where the single-pass damping is weak. Only AORSA includes MC rigorously via its all orders formulation whereas EVE, PSTELION and TORIC include MC via reduced formulation. However, the AORSA simulation did not use sufficiently high resolution to resolve mode converted waves in ITER. In cases such as this where the cyclotron layer is far off axis, (see location in table 4), high resolution is required. AORSA-iso and CYRANO results are especially low for the thermal $\mathrm{H}$ partitions.

The perpendicular and parallel energy densities of the minority ions specified for the wave-solvers are evolved in PTRANSP by FPPRF and are input to the full-wave solvers as effective tail temperatures. The AORSA-CQL3D result (using CQL3D for the minority distribution) is shown for comparison in the tables. It is known that use of effective Maxwellian distributions are adequate for fundamental resonance heating $[27,28]$, but can over-estimate the high energy tail for cases with second harmonic absorption. This can get exaggerated as TORIC iterates with FPPRF. CQL3D can predict the $\mathrm{He}^{3}$ phase-space distribution including the second harmonic $\mathrm{He}^{3}$ cyclotron damping without assuming a form for the minority velocity distribution. The differences in results in table 8 between PTRANSP-FPPRF and AORSA-CQL3D, and even between AORSA-CQL3D and AORSA-iso could likely be a consequence of using an effective Maxwellian to treat the second harmonic absorption. Much closer agreement is found for the $\mathrm{He}^{3}$ absorption in case 3, shown in table 9, although CYRANO is relatively high for the electron partition. The better agreement among codes for case 3 relative to case 2 is consistent with higher single-pass absorption that occurs in $\mathrm{H}$-mode parameters chosen for this case (see figure $2(d)$ ).

On the other hand the TORIC-FPPRF and AORSACQL3D predictions for fundamental absorption in cases 1 and 4 are much closer, suggesting the simpler treatment of fitting the energetic tail to mono- (iso-) or bi-Maxwellians may be a better approximation for the stronger single-pass fundamental minority heating cases, or that the FLR approximation used in the full-wave solvers (except for AORSA) is more accurate than for the 2nd harmonic $\mathrm{He}^{3}$ scenario.

Contours of components of the electric field are shown in figures 4 and 5. These show qualitative agreement for the fullwave solutions, and confirm the weak-single-pass absorption shown in figure 3. Contours of ICRF heating for case 1 are shown in figure 6. 

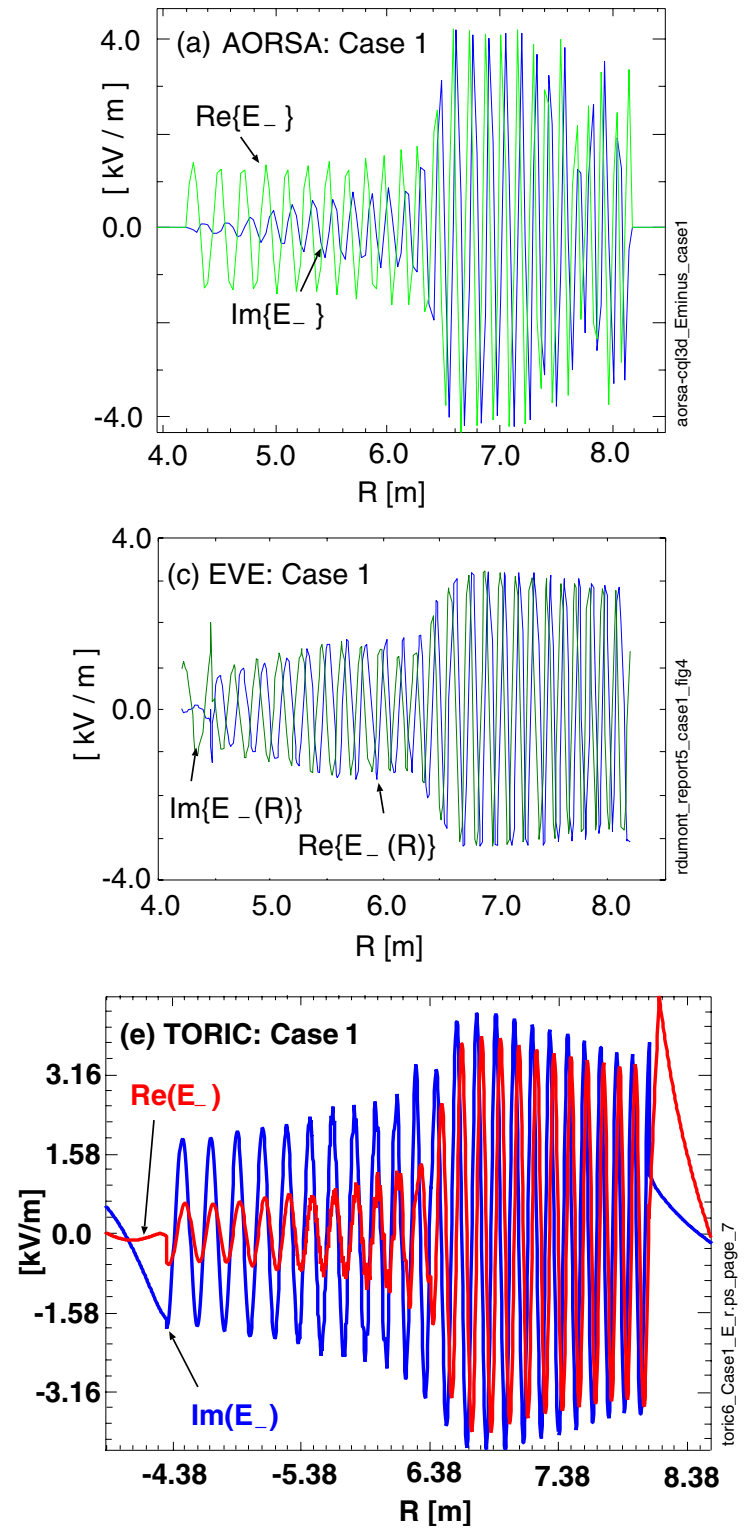
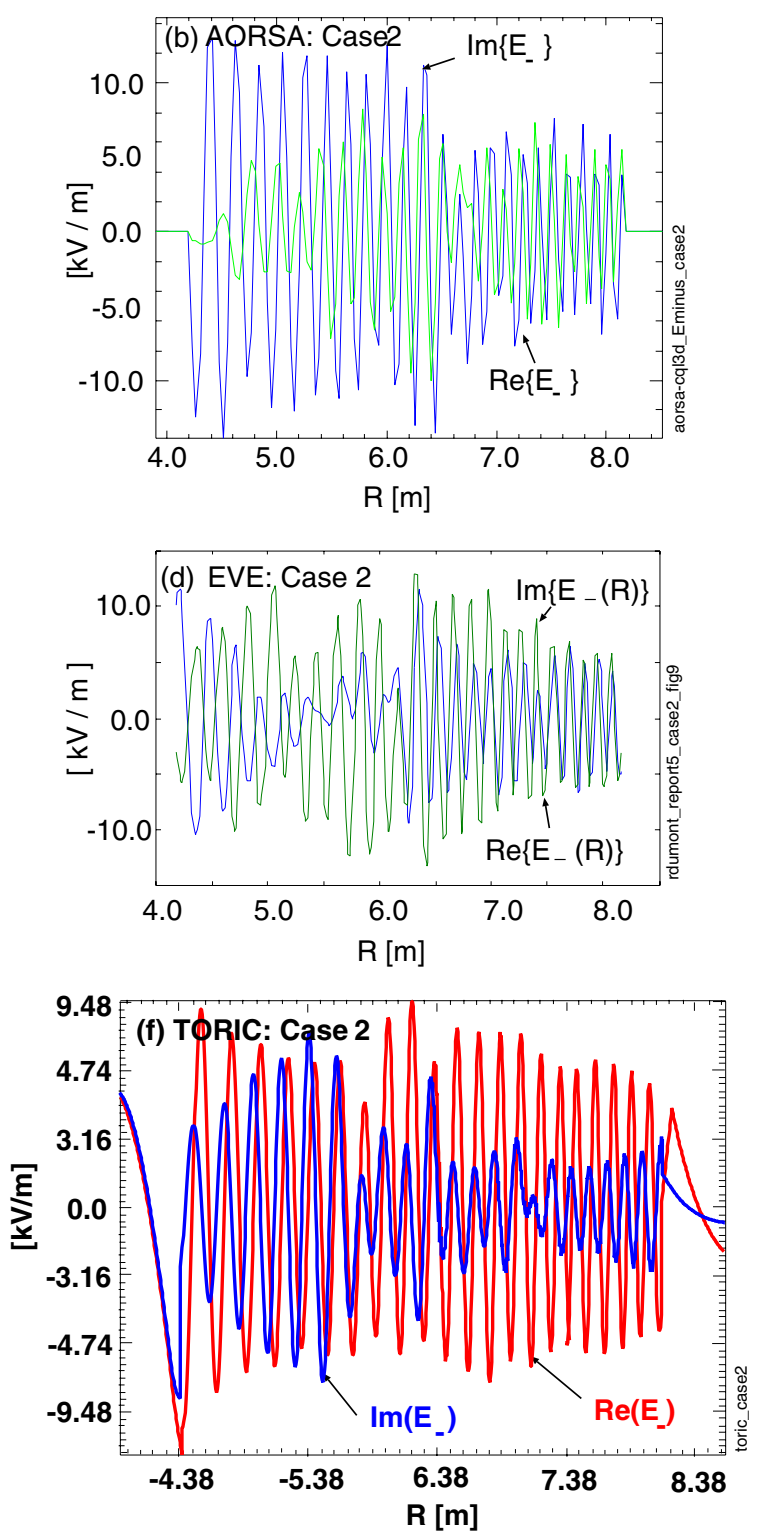

Figure 3. Profiles along the midplane at the height of the magnetic axis of the real and imaginary parts of $E_{-}$for cases 1 (with fundamental resonance $\mathrm{He}^{3}$ ) and 2 (with second harmonic $\mathrm{He}^{3}$ ) from $(a)-(b)$ AORSA-CQL3D simulations, $(c)-(d)$ EVE, and $(e)-(f)$ TORIC (v6). The magnetic axis is near $6.38 \mathrm{~m}$.

Table 8. Comparisons of heating partitions (\%) for pre-activation bulk $\mathrm{H}$ case 2 with $n_{\mathrm{He}^{3}} / n_{\mathrm{e}}=0.03$. The PTRANSP-aniso and TORIC-iso partitions to electrons are split into FW and IBW.

\begin{tabular}{llllll}
\hline Code & $\mathrm{He}^{3}$ & Electrons & $\begin{array}{l}\text { Thermal } \\
\mathrm{H}\end{array}$ & $\begin{array}{l}\text { Beam } \\
\mathrm{H}\end{array}$ & $\begin{array}{l}\text { Carbon } \\
\text { impurity }\end{array}$ \\
\hline PTRANSP-aniso & 29.1 & $42.1+7.0$ & 21.4 & 0.4 & 0.0 \\
AORSA-iso & 23.0 & 69.4 & 7.5 & N.A. & 0.03 \\
CYRANO-iso & 31 & 64 & 5 & N.A. & $<0.1$ \\
EVE-aniso & 17.2 & 68.2 & 13.5 & 1.2 & 0.0 \\
EVE-iso & 15.6 & 68.9 & 14.5 & 1.0 & 0.0 \\
TORIC-iso & 9.7 & $66.3+11.3$ & 12.1 & 1.0 & 0.0 \\
AORSA-CQL3D & 12.4 & 78.4 & 9.0 & N.A. & 0.03 \\
\hline
\end{tabular}

The heating predictions required for use in present stateof-art transport codes are flux-surface averaged profiles. Simulations of direct ICRF-heating profiles and their volume integrals for case 1 are shown in figure 7 . Qualitative
Table 9. Comparisons of heating partitions (\%) for pre-activation bulk $\mathrm{H}$ case 3 with $n_{\mathrm{He}^{3}} / n_{\mathrm{e}}=0.20$. The PTRANSP partition to electrons is further split into fast wave and IBW when available.

\begin{tabular}{llllll}
\hline Code & & & $\begin{array}{l}\text { Thermal } \\
\text { Beam }\end{array}$ & $\begin{array}{l}\text { Carbon } \\
\text { impurity }\end{array}$ \\
\hline PTRANSP-aniso & 76.3 & $17.1+1.4$ & 4.5 & 0.7 & 0.0 \\
AORSA-iso & 79.1 & 18.8 & 2.1 & N.A. & 0.1 \\
CYRANO-iso & 51 & 45 & 4 & N.A. & $<0.1$ \\
EVE-aniso & 45.2 & 37.7 & 9.2 & 7.9 & 0.0 \\
EVE-iso & 58.1 & 30.0 & 5.6 & 6.3 & 0.0 \\
TORIC-iso & 53.6 & $36.7+1.1$ & 7.7 & 0.85 & 0.0 \\
AORSA-CQL3D & 64.0 & 30.0 & 5.9 & N.A. & 0.09 \\
\hline
\end{tabular}

agreement is found for most of the benchmark full-wave solvers. The PSTELION results, shown in figure $7(d)$, are broader than the others, apparently due to the use of insufficient numerical resolution or approximate equilibrium. CYRANO 

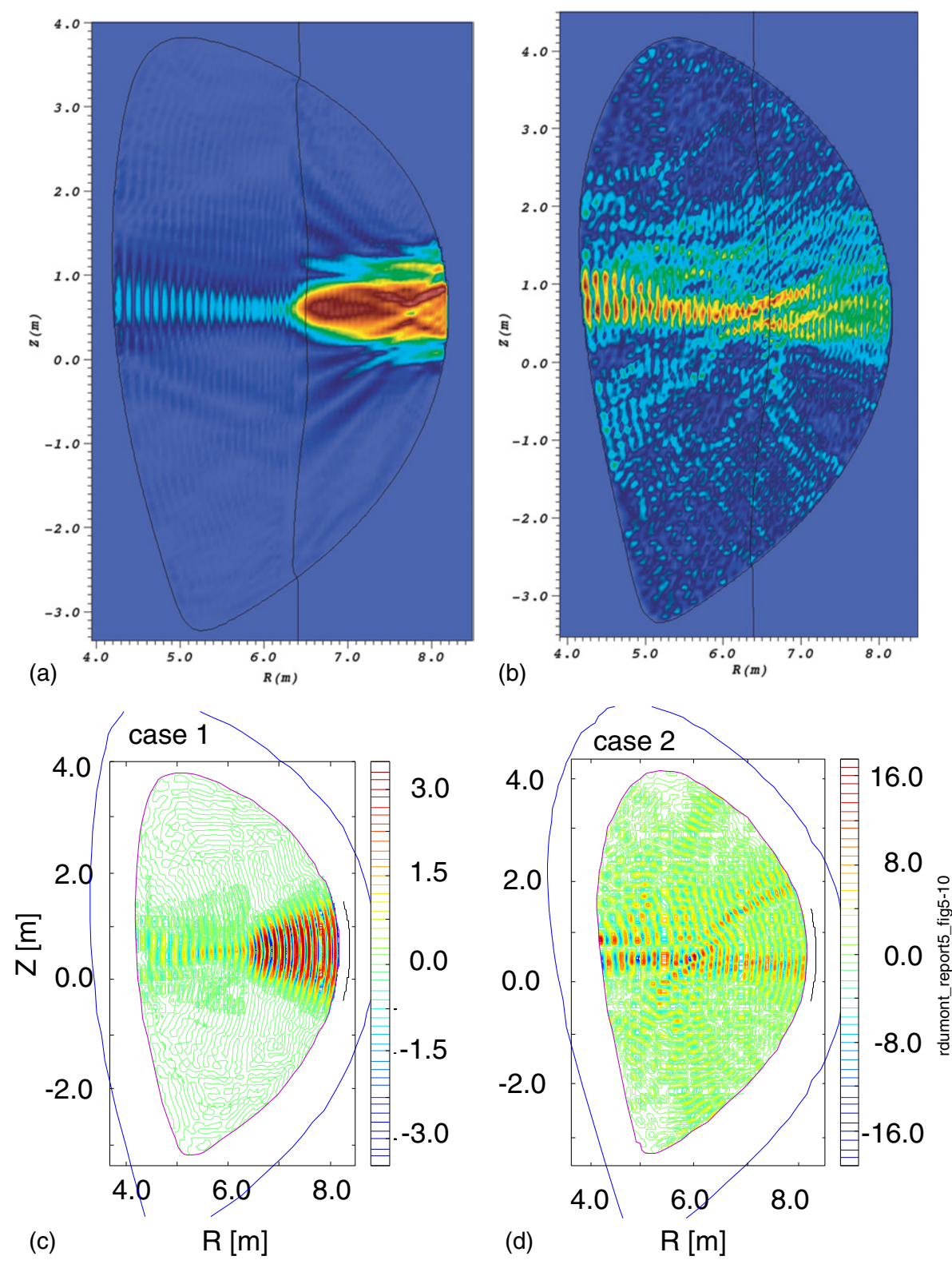

Figure 4. Contours of the real part of components of $\operatorname{Re}\left[E_{-}\right]$for cases 1 and 2 simulated by $(a)$ AORSA case 1 ; $(b)$ AORSA case 2 ; $(c)$ EVE-iso for case $1 ;(d)$ EVE-iso for case 2. Single-pass absorption is stronger for case 1.

and EVE have similar profiles for the pre-activation case 4 with bulk $\mathrm{He}^{4}$ shown in figure 8 . Results are summarized in table 7 .

Profiles of ICRF-driven currents are also needed for realistic modelling. These can be calculated using fullwave solvers coupled to Fokker-Planck solvers, or using the Ehst-Karney [29] formula which is a parameterization of the current drive efficiency obtained from an adjoint calculation. Examples from three of the full-wave solvers using this formula are shown in figure 9. The results from AORSA-iso are similar to results from AORSA-CQL3D (not shown). The fluxsurface (area-integrated) currents are also shown. The currents simulated for the pre-activation cases (with half current) are two to six times that for the DT case 1 . For case 2 the total ICRF-driven current RFCD is $0.7 \mathrm{MA}$ which is significant compared with the total plasma current of 7.5 MA, so effects on the $q$ profile and equilibrium could be significant. Thus RFCD could be useful for controlling the current, especially in the core plasma where the electron temperature is highest and the effects of particle trapping are small. The results from EVE and TORIC for case 1 are also shown. The total simulated currents are slightly higher than that in figure $9(a)$. The TORIC peak current profile near the axis in figure $9(f)$ is higher by a factor of 2.5. These differences could be due mainly to differences in grid resolution. The total RFCD currents are comparable to those in figure 9. The indirect plasma current drive from the minority ions is not included.

\section{Results for variations of the cases with reduced minority concentrations}

There are several motivations for extending the studies to cases for which the minority fractions are reduced. For instance, 

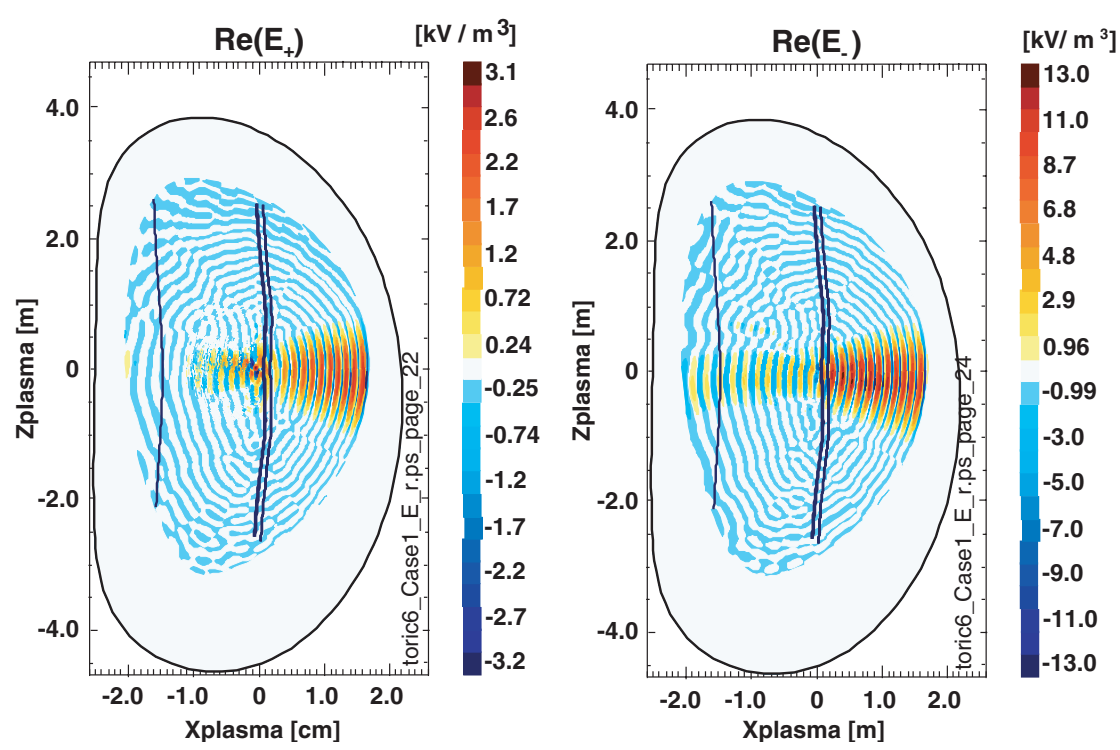

Figure 5. TORIC simulations of contours of $\operatorname{Re}\left[E_{+}\right]$and $\operatorname{Re}\left[E_{-}\right]$for case 1 , in units of $\left(\mathrm{kV} \mathrm{m}^{-3}\right)$. The major radius and vertical displacements are measured relative to the magnetic axis shown in figure 1.
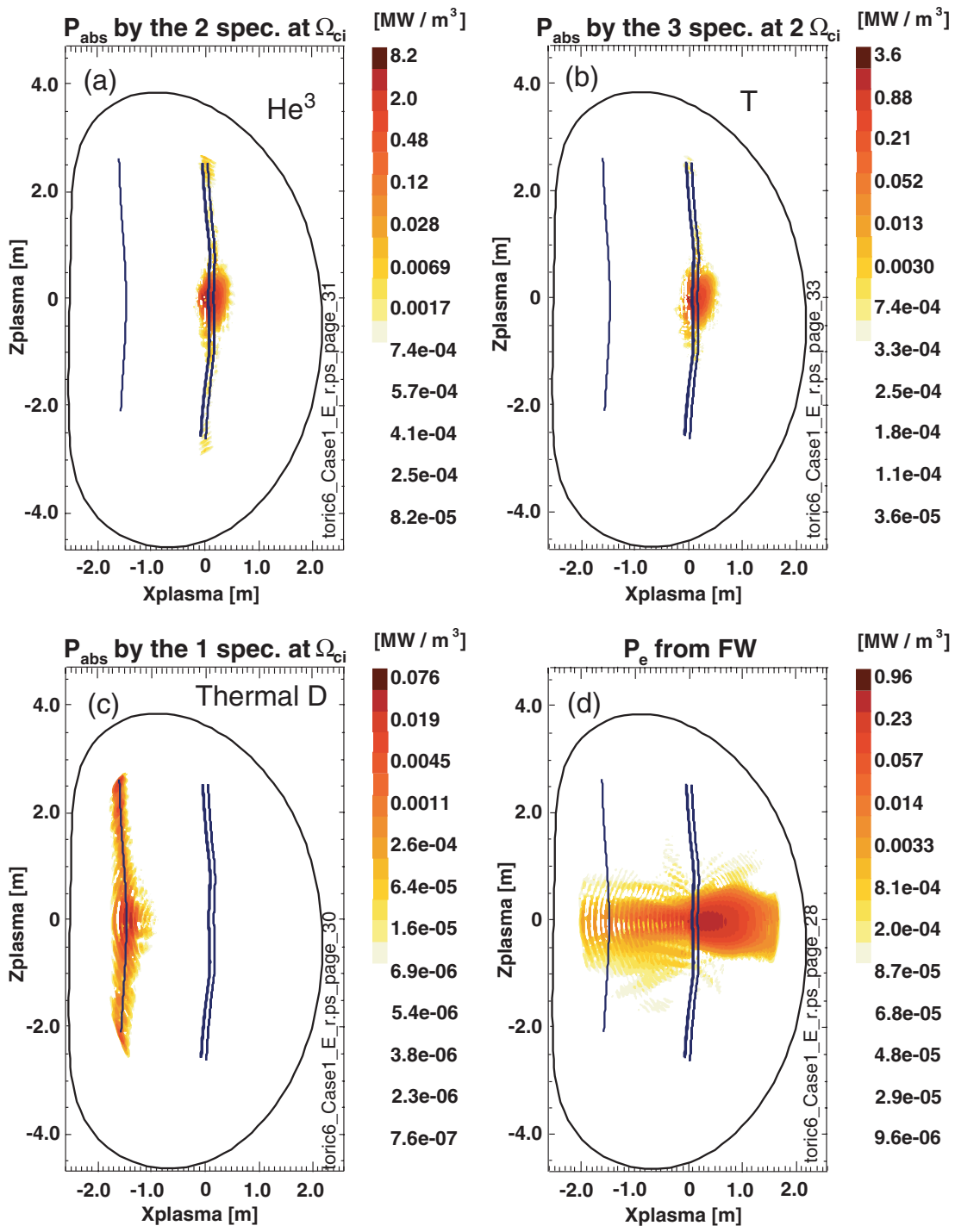

Figure 6. TORIC simulations of contours of power deposited for case 1 in units of $\left(\mathrm{MW} / \mathrm{m}^{-3}\right)$. 

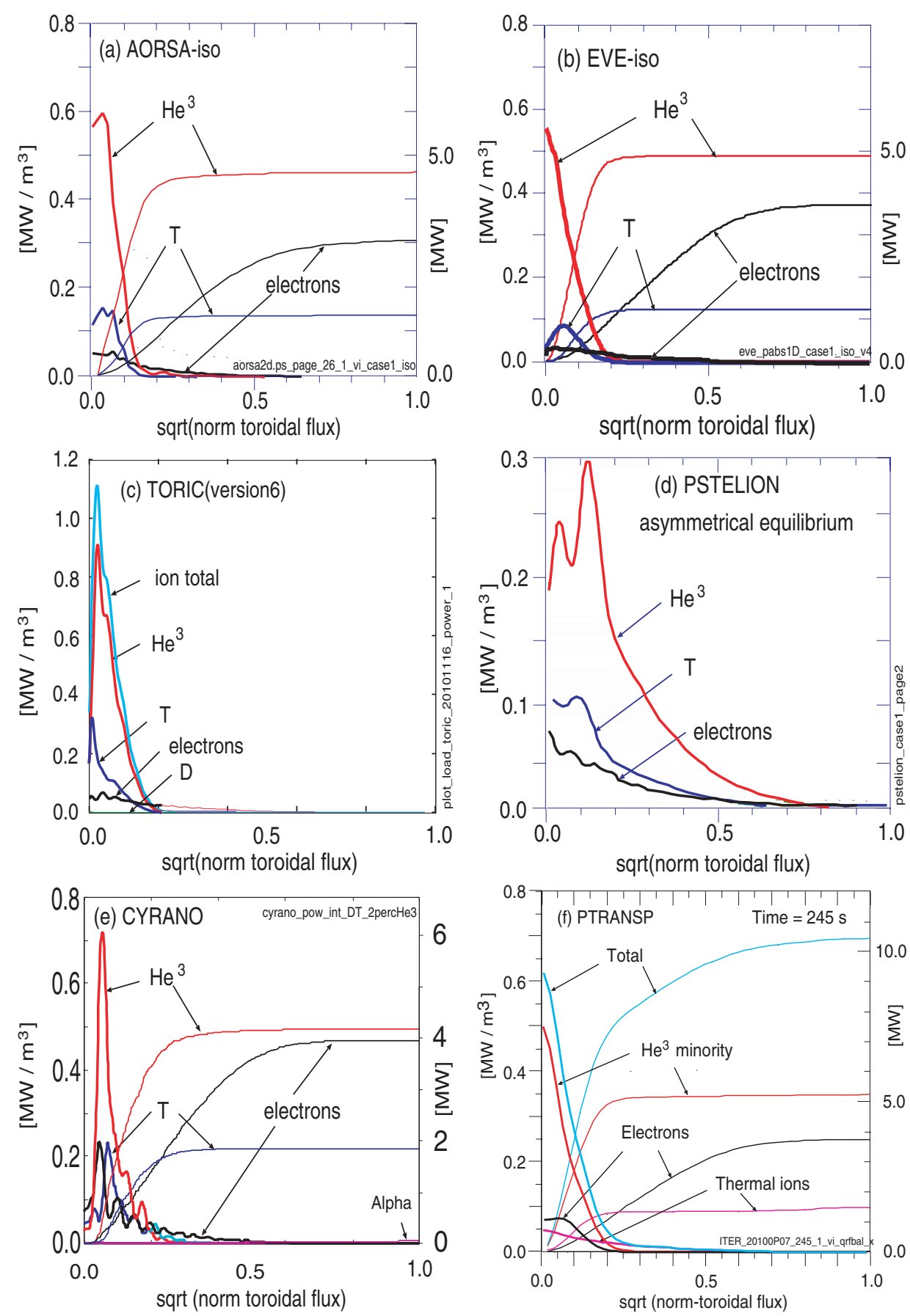

Figure 7. Simulations of the direct RF-plasma heating power density profiles for the DT case 1 (with $2 \% \mathrm{He}^{3}$ ).

$\mathrm{He}^{3}$ is expensive so routine use at high concentrations might be unacceptable. Variations in the fraction of minority species are studied with some of the full-wave solvers. The results for the heating partitions for case 1 are in general agreement with the fraction of tritium heating increasing to $\simeq 40-50 \%$ and the fraction to the minority decreasing to low values as the fraction of minority ion density decreases. Comparisons of the heating partitions with an alternative assumption of low $\mathrm{He}^{3}$ fraction are given in table 10. The 1D heating profiles computed by EVE for a case with $n_{\mathrm{He}^{3}} / n_{\mathrm{e}}=0.001$ are shown in figure $10(b)$. A large fraction is predicted to be deposited on the argon impurity.
It is interesting to note that the electron absorption for the two cases is about the same, but that the $T$ power is reduced by about the same amount as the $\mathrm{He}^{3}$ minority increases. Since some of the $\mathrm{He}^{3}$ minority power is deposited on the electrons (via slowing down), it appears that the limiting case of zero $\mathrm{He}^{3}$ could offer slightly more direct ion heating than the minority case.

\section{Grids and convergence}

The numerical grid needs to be sufficiently fine to achieve accurate solutions. For instance, a fine grid is needed to 

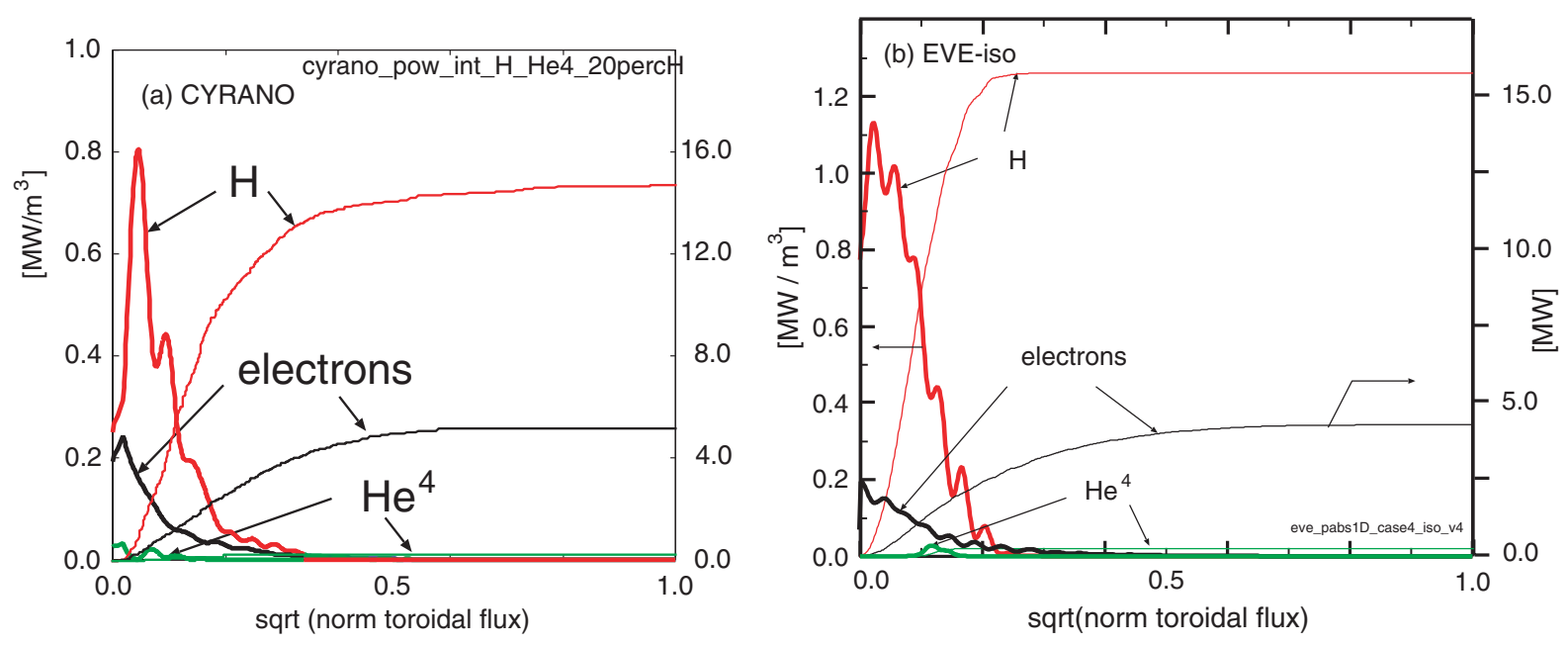

Figure 8. Simulations of the direct RF-plasma heating power density profiles for the bulk $\mathrm{He}^{4}$ case 4 (with $20 \% \mathrm{H}$ ) from $(a)$ CYRANO and (b) EVE. The heating partitions are shown in table 7.

accurately localize the ion-ion and cyclotron resonance layers, and to resolve $\mathrm{MC}$, and small wavelength phenomena. Various checks indicate how well converged the solutions are. One test of the accuracy of the global power balance is given by comparing the antenna loading with the Poynting flux through the plasma surface, and the total power deposited in the plasma. Another check is given by comparing the radial oscillations of the electric field with the grid spacing. Still another is by checking for curvature in the cyclotron layer. Curvature indicates insufficient poloidal resolution.

Initial AORSA runs were conducted with a $(R, Z)$ grid of $128 \times 128$ (in cylindrical $R, Z$ space) for the Fourier basis sets. These results were checked increasing the number of grids to $256 \times 256$. This gave little change in the results for the heating partitions, although this resolution is still too coarse to resolve $\mathrm{MC}$ to ion-cyclotron waves. $\mathrm{A}(R, Z)$ grid of about $500 \times 500$ would be needed. Runs with $256 \times 256$, require $\simeq 1000$ processor-hours (e.g. 1 hour on 1024 processors), while the $128 \times 128$ runs required $\simeq 4^{3}$ less resources (a few minutes at 512 processors). This CPU scales as $n^{3}$ in the resolution with a grid of $n \times n$.

Results from a TORIC study of convergence as the grid resolution is refined is given in table 11. The TORIC simulations for the heating partitions are well converged with 63 poloidal modes and 403 radial zones, but resolution of IBW requires 127 poloidal modes. The TORIC convergence is discussed in more detail in appendix B.6. The PTRANSP runs used 31 poloidal modes which are insufficient for resolving MC. These runs used hundreds of CPU-hours for the TORIC part of the (time-dependent) calculation on one processor. The TORIC CPU scales as the number of poloidal modes cubed. PTRANSP-TORIC has recently been parallelized to facilitate running with more poloidal modes.

\section{Discussion and conclusions}

A benchmarking study of six ICRF solvers has been carried out using four ITER scenarios that were obtained from integrated modelling predictions. The benchmarking simulations performed for this study find small heating powers damped by the impurity and fast-ion species. This suggests that simulations might obtain accuracy without including many ion species, or alternatively that each ion impurity could be included as a single fully stripped species. Numerical convergence for case 1 is studied using AORSA, EVE and TORIC. Results show that for cases with strong single-pass damping (cases 1 and 4) good convergence in the solutions is achieved with grids compatible with integrated, time-dependent prediction codes. Comparisons of the assumptions of mono-Maxwellian versus bi-Maxwellian minority temperatures are done using EVE for all four cases. The results show small (few\%) effects in the heating fractions.

The benchmarking of full-wave solvers for the fundamental harmonic cases 1 and 4 give similar results for some of the solvers, indicating that reliable solutions can be achieved. One open question concerning case 4 is whether MC plays a significant role with the high minority fraction assumed $(20 \% \mathrm{H})$. If the radial/poloidal grid is sufficiently coarse the simulation will not capture the short wavelength structure. It may be that $\mathrm{MC}$ is really not efficient because of the large gap between the fast wave (FW) cutoff and the MC layer in this case.

Results for the pre-activation second harmonic cases 2 and 3 with bulk $\mathrm{H}$ have larger differences among the code predictions. Weak-single-pass damping at the second harmonic $\mathrm{He}^{3}$ layer can lead to differences in simulation results as wave fields are reflected multiple times at the plasma boundary. In these cases where multi-pass damping is present it may be useful to perform simulations with multiple $k_{\|}$ components coupled by the antenna since these components can each behave differently. In the final analysis, these cases might not be useful for ITER due to the cost of $\mathrm{He}^{3}$.

One of the most important areas that needs to be addressed in future studies are the reasons for differences in code predictions, especially in cases of weak-single-pass damping. Numerical convergence studies such as that performed with the AORSA, TORIC and EVE solvers should be done with the other solvers. In addition the use of more complete antenna spectra (more toroidal wavenumbers) should be investigated. In order to further understand the role of different MC models in the solvers it would also be useful to perform 'gedanken' 

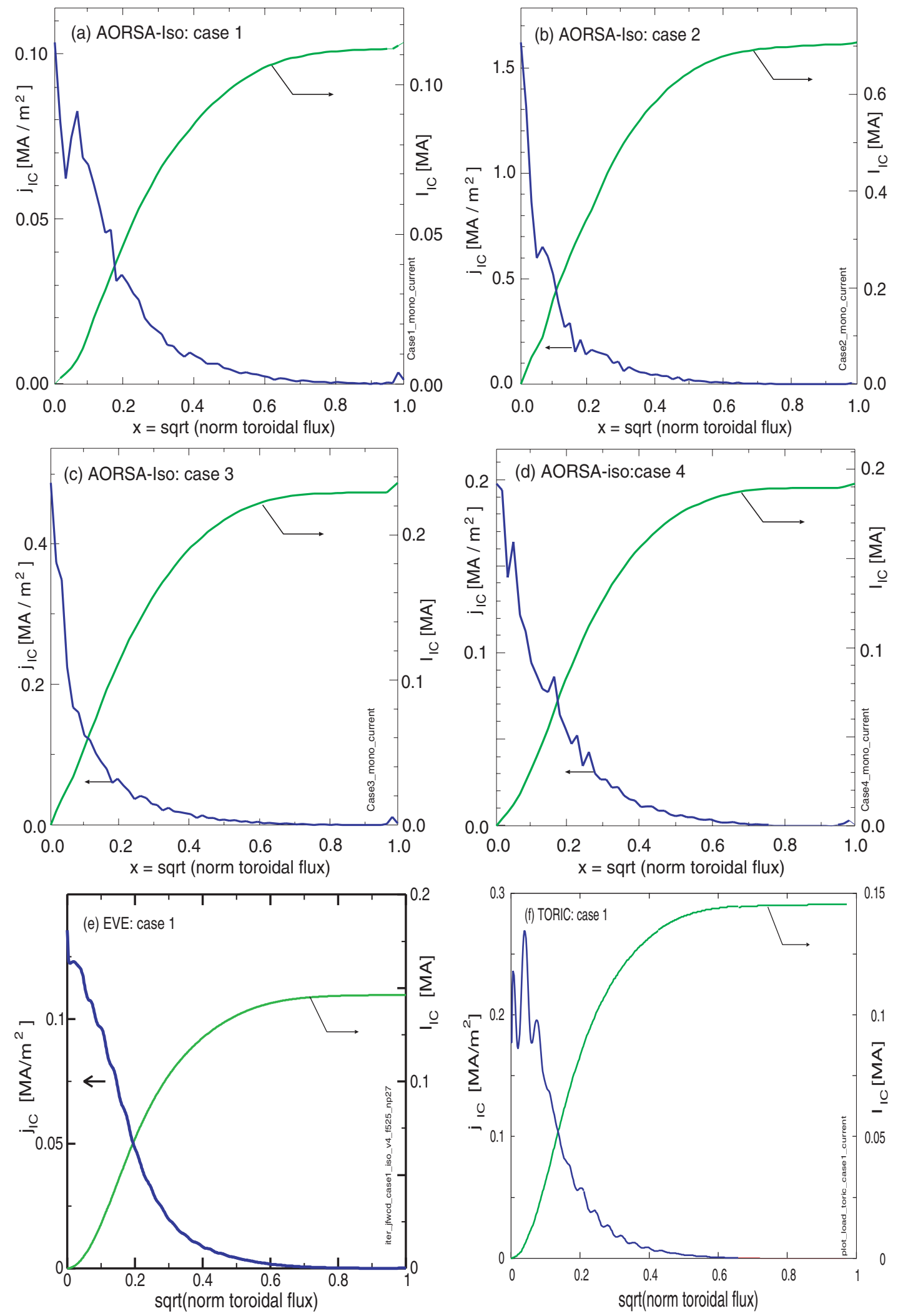

Figure 9. AORSA-Iso, EVE and TORIC-v6 simulations of ICRF-driven current profiles and their area-integrals.

simulations in which the role of MC is eliminated, such as a deuterium majority and hydrogen minority $(\simeq 5 \% \mathrm{H})$ ITER discharge. In this case one might expect substantial agreement among all the codes.
In conclusion, the present ICRF benchmark study has yielded important information concerning differences and similarities that one can expect amongst a group of fullwave solvers. This information is extremely useful as it 
Table 10. Comparisons of heating partitions (\%) for the DT case 1 as the $\mathrm{He}^{3}$ fractions are reduced. For the EVE results, due to the presence of the Alfvén resonance wave layer on the high-field side which results in spurious damping of the wave by the argon, the power split is estimated at normalized radius $=0.9$. At $\mathrm{He}^{3} / n_{\mathrm{e}}=0.001$ damping on the argon is predicted by EVE to be significant, shown in figure $10(b)$.

\begin{tabular}{|c|c|c|c|c|c|c|c|c|c|c|}
\hline Full-wave solver & $\mathrm{He}^{3} / n_{\mathrm{e}}$ & Tritium & Deuterium & Minority & Ash & Electrons & Argon & Beryllium & D-beams & Alphas \\
\hline \multirow[t]{2}{*}{ AORSA-CQL3D } & $2 \%$ & 15 & 0.9 & 50 & 0.3 & 33 & 0.2 & 0.3 & 0 & 0 \\
\hline & $0.2 \%$ & 51 & 2 & 9 & 0.1 & 36 & 0 & 0 & 0.5 & 1.7 \\
\hline \multirow[t]{4}{*}{ CYRANO } & $3 \%$ & 14 & 0 & 46 & NA & 39 & NA & NA & NA & 1 \\
\hline & $2 \%$ & 18 & 1 & 41 & NA & 39 & NA & NA & NA & 1 \\
\hline & $1 \%$ & 25 & 1 & 27 & NA & 46 & NA & NA & NA & 1 \\
\hline & $0 \%$ & 38 & 1 & 0 & NA & 60 & NA & NA & NA & 1 \\
\hline EVE-aniso & $2 \%$ & 12.9 & 0.4 & 52.8 & 0.1 & 39.1 & 1.4 & 0.2 & 0.0 & 0.2 \\
\hline EVE-iso & $2 \%$ & 13.5 & 0.5 & 51.7 & 0.1 & 32.2 & 1.6 & 0.3 & 0.0 & 0.2 \\
\hline EVE-aniso & $0.1 \%$ & 44.2 & 3.1 & 14.1 & 0.2 & 37.9 & 0.0 & 0.0 & 0.2 & 0.4 \\
\hline EVE-iso & $0.1 \%$ & 44.8 & 3.2 & 12.9 & 0.2 & 38.5 & 0.0 & 0.0 & 0.1 & 0.4 \\
\hline
\end{tabular}
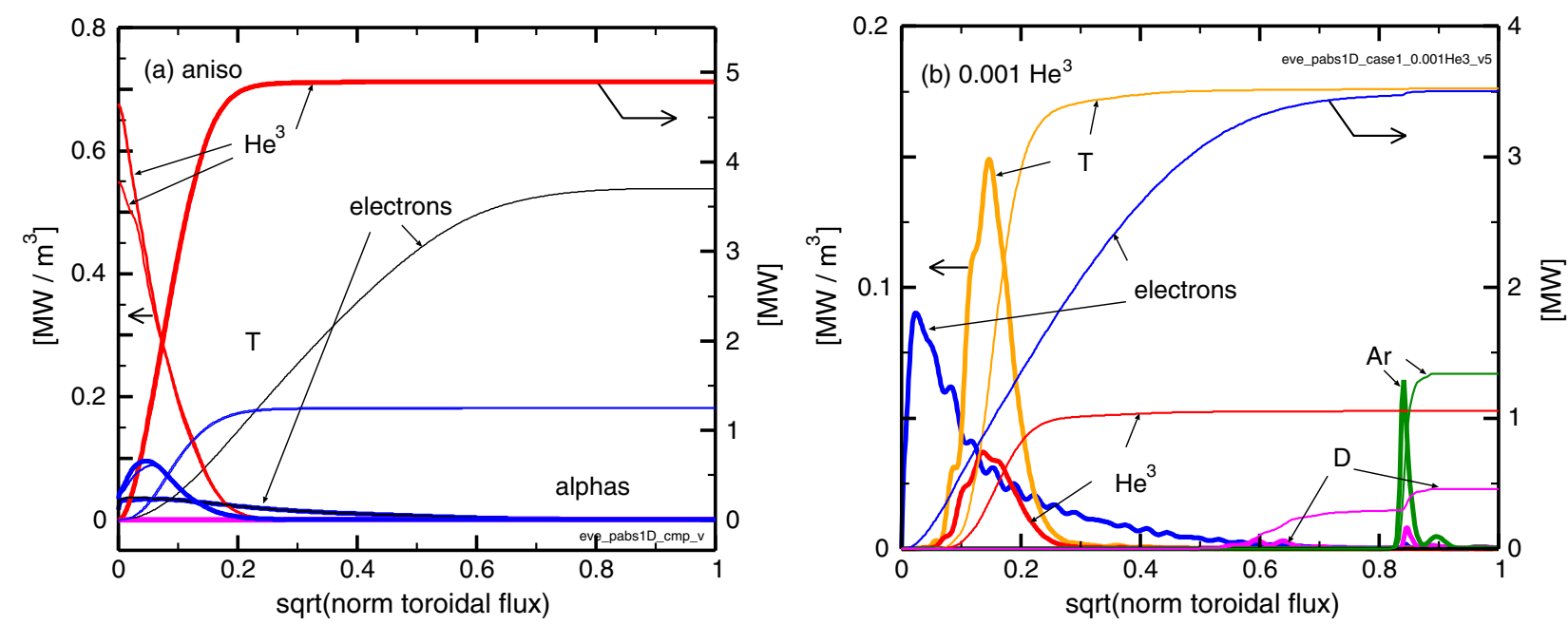

Figure 10. Simulations of heating power profiles for the DT cases 1 from EVE. $(a)$ Comparison of isotropic (dark) (figure 7(b)) and anisotropic (light) temperatures. Small differences near the magnetic axis are seen; $(b)$ results of lowering $n_{\mathrm{He}^{3}} / n_{\mathrm{e}}$ from 0.02 to 0.001 The wave is not well absorbed after the first pass. Part of absorption on D and all for the Ar occurs near the plasma edge. The tritium heating increases from $15 \%$ to $35 \%$.

Table 11. Results from a TORIC convergence scan for heating partitions $(\%)$ for the DT case 1 , at an earlier time in the simulation (149s).

\begin{tabular}{llllll}
\hline $\begin{array}{l}\text { Poloidal } \\
\text { modes }\end{array}$ & $\begin{array}{l}\text { Radial } \\
\text { points }\end{array}$ & $\begin{array}{l}\text { 1st } \\
\text { harmonic } \mathrm{T}\end{array}$ & $\begin{array}{l}\text { Fundamental } \\
\mathrm{He}^{3}\end{array}$ & $\begin{array}{l}\text { el fast } \\
\text { wave }\end{array}$ & $\begin{array}{l}\text { el } \\
\text { IBW }\end{array}$ \\
\hline 31 & 203 & 12.44 & 58.65 & 27.41 & 0.20 \\
31 & 403 & 12.36 & 58.71 & 27.20 & 0.20 \\
63 & 403 & 13.12 & 61.18 & 24.50 & 0.16 \\
127 & 403 & 13.26 & 61.00 & 24.48 & 0.25 \\
127 & 803 & 13.23 & 61.10 & 24.42 & 0.24 \\
255 & 803 & 13.04 & 61.12 & 24.71 & 0.27 \\
\hline
\end{tabular}

informs the application of these solvers in integrated modelling studies.

\section{Acknowledgments}

The authors wish to thank P. Lamalle, A. Polevoi and J. Snipes for helpful suggestions and comments. This work is supported in part by the US DoE Contract No DE-ACO2-76-CHO3073.

\section{Appendix A. PTRANSP predictions of the benchmark cases}

The PTRANSP code [5-8] is used to generate predictions for the plasma conditions. The predictions are integrated and self-consistent in that the heating, current drive and beam torques are calculated using predicted plasma profiles. Physics effects not yet included in PTRANSP are the ICRFinduced and intrinsic rotation. The heating, neutral-beamtorquing and current-drive profiles are used in the local flux-averaged energy, momentum and magnetic field balance equations, and the time-evolution of the temperature and minority distributions is predicted. The up/down asymmetric geometry of the flux surfaces are included. An example is shown in figure 1 . The temperatures are calculated using GLF23 [30] and a pedestal model [31] incorporated into PTRANSP which predicts the pedestal pressure. The boundary values for GLF23 are the temperatures at the top of the pedestal. Temperature profiles are shown in figure 2. For case 1 the toroidal rotation profile (needed for the flow-shearing rate) is calculated assuming that the ratio of momentum to ion energy transport is 0.5 . For the other cases GLF23 is used to also compute self-consistently the toroidal rotation. 

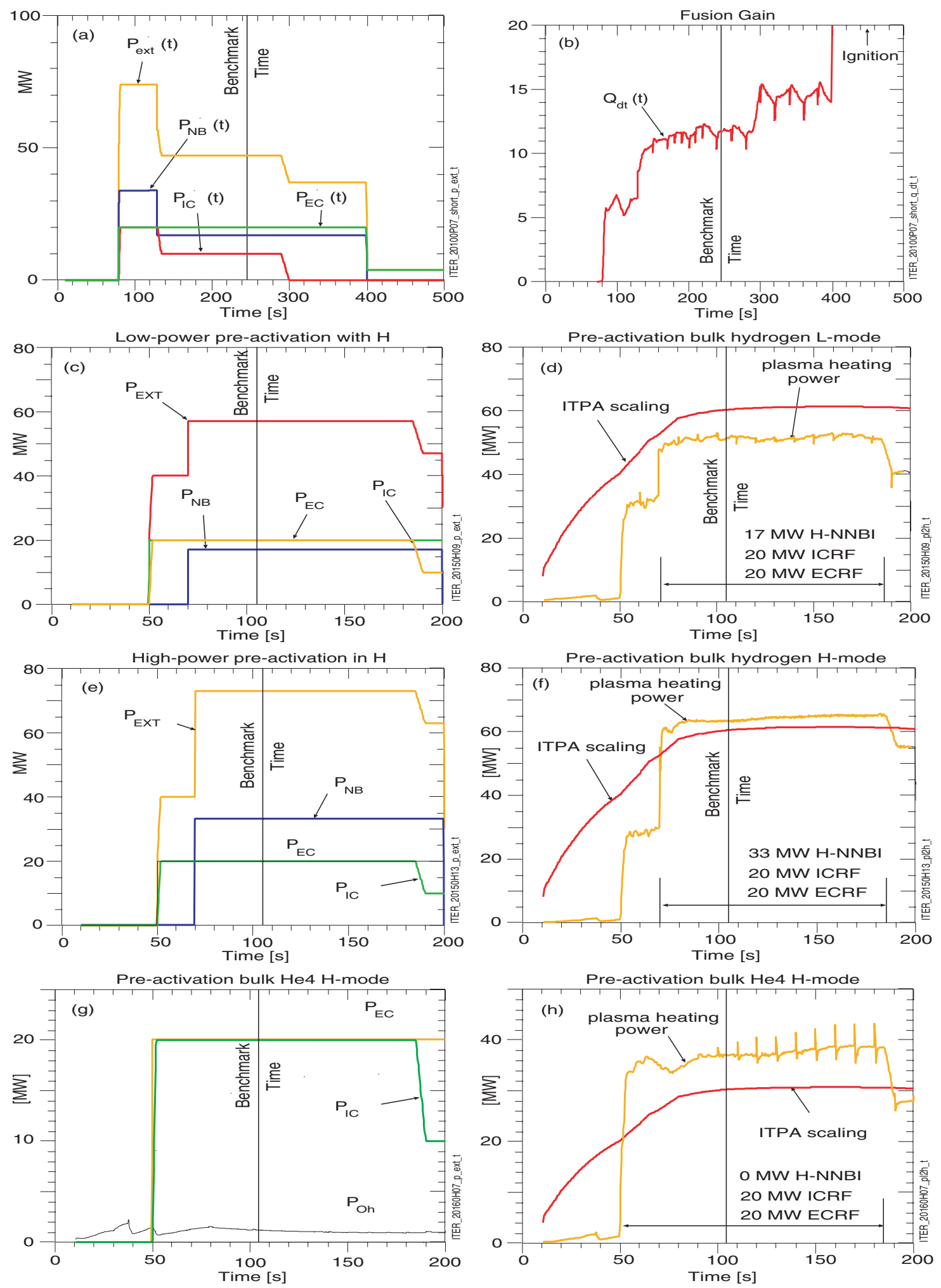

Figure 11. Waveforms from PTRANSP predictions. The times of the benchmark cases are indicated by vertical lines.

The plasma current and toroidal field are assumed to be $15 \mathrm{MA}$ and 5.3 T. The electron density profile is assumed to be flat and ramped to a Greenwald fraction of 0.85 . The auxiliary heating for the DT case 1 is assumed to start with $73 \mathrm{MW}$ (the total planned for ITER), since the maximum may be needed to induce the transition to a high-performance H-mode. This power is composed of $33 \mathrm{MW}$ of D-NNBI at $1 \mathrm{MeV}, 20 \mathrm{MW}$ ICRF and $20 \mathrm{MW}$ ECRF. The heating is stepped down as the alpha heating increases, thereby allowing the fusion gain $Q_{\text {DT }}$ defined as the ratio of the fusion yield over the auxiliary and ohmic powers to increase. The heating power evolutions are shown in figure $11(a)$. The $\mathrm{Q}_{\mathrm{DT}}$ evolution is shown in 

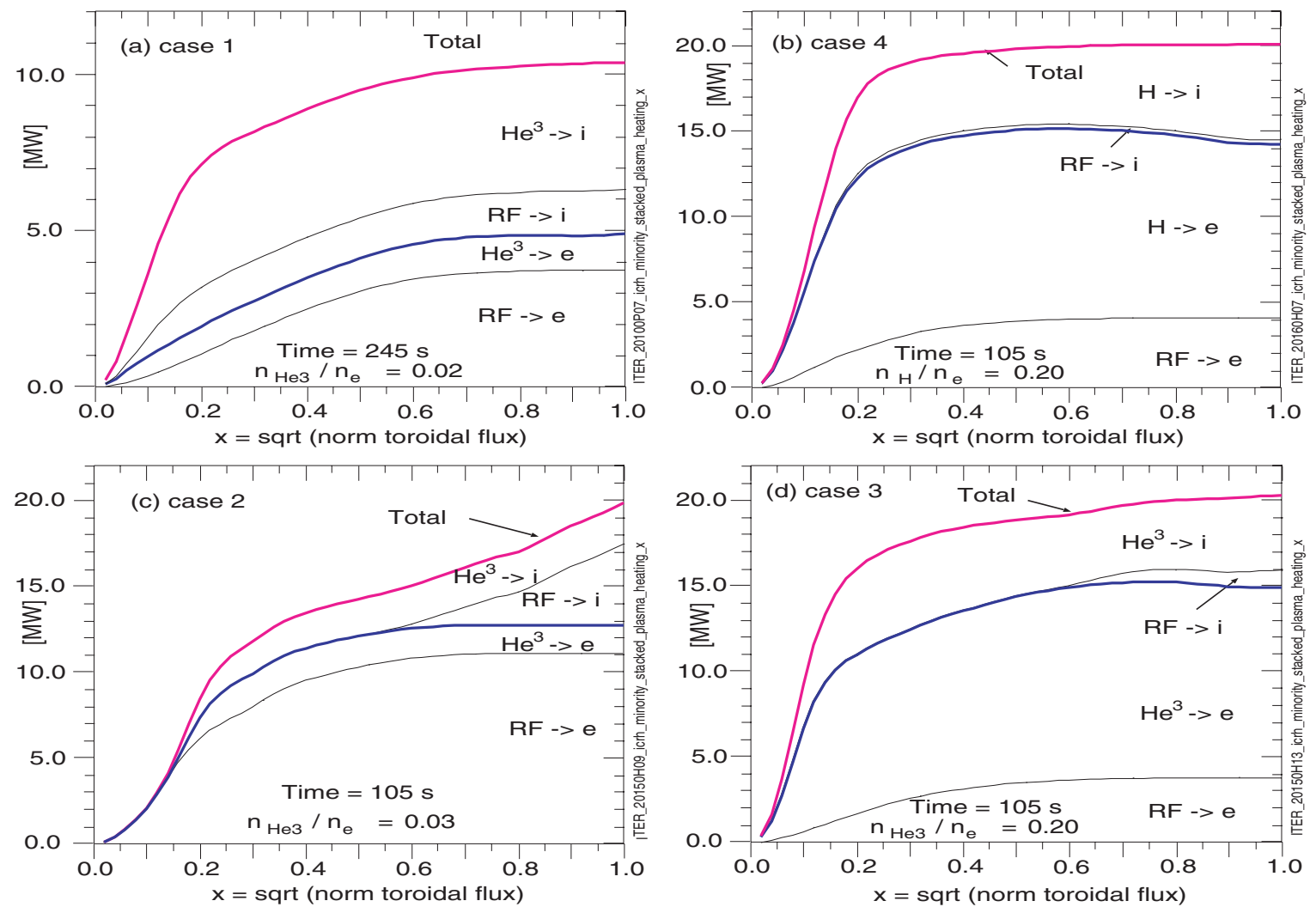

Figure 12. Stacked ICRF and minority heating profiles for the benchmark cases, computed from PTRANSP-FPPRF. For case 1 about half of the ICRF power (direct heating and minority slowing down) is predicted to be deposited on thermal ions. For the other cases the fraction is less.

figure 11(b). The assumed thermal ion species are $\mathrm{D}, \mathrm{T}, \mathrm{He}^{4}$ ash, Be and Ar impurities; the fast-ion species are D-beams, and alphas. The electron density $n_{\mathrm{e}}$ is assumed to be flat, and the $\mathrm{Be}$ and $\mathrm{Ar}$ densities are assumed to be $n_{\mathrm{Be}} / n_{\mathrm{e}}=0.02$ and $n_{\mathrm{Ar}} / n_{\mathrm{e}}=0.0012$. The gas fuelling, recycling, and ash transport are described in [7]. The plasma has $Q_{\mathrm{DT}} \simeq 12$ at the benchmarking time (245 s).

The PTRANSP runs typically use several hundreds of hours of CPU to model an $800 \mathrm{~s}$ ITER H-mode. Of this $32 \mathrm{~h}$ are used to simulate the ICRF heating lasting $220 \mathrm{~s}$ using TORIC (version 5) with a low number of poloidal modes (31). The runtime increases as the cube of the number of poloidal modes, so increased accuracy requires much more CPU.

The pre-activation plasmas are assumed to have a shorter duration with the $n_{\mathrm{e}}$ profile ramped up to a peak of $4.6 \times$ $10^{19} \mathrm{~m}^{-3}$ by $80 \mathrm{~s}$. The electron density profile is assumed to be flat and ramped to a Greenwald fraction of 0.75 . The profile is assumed to be flat. ICRF heating of $20 \mathrm{MW}$ and ECRF heating of $20 \mathrm{MW}$ are assumed to start at $50 \mathrm{~s}$. For the bulk $\mathrm{H}$ cases 2 and 3 the H-NNBI is assumed to be $17 \mathrm{MW}$ and $33 \mathrm{MW}$, respectively. The evolutions of the heating powers are shown in figure 11. The beam voltage is assumed to be $870 \mathrm{keV}$ to avoid excessive power shine-through. The impurity is assumed to be only C with density $2 \%$ of $n_{\mathrm{e}}$. GLF23 is run in the 'option 2' mode discussed in [8] with the toroidal rotation and flow-shearing rate profiles computed by GLF23. This results in predictions achieving moderately high central temperatures even with low pedestal temperatures.

Recent extrapolations of an ITPA database values of the $\mathrm{L} \rightarrow \mathrm{H}$ power threshold scalings of the $\mathrm{H}$-mode threshold indicate that the case 2 with $17 \mathrm{MW}$ H-NNBI will not achieve the H-mode (figure $11(c)$ ), but case 3 with $33 \mathrm{MW}$ will (figure 11(e)). The threshold power increases with density and toroidal field, so lower density and toroidal field are preferable. Comparisons of the predicted volume-integrated thermal ion and electron heat depositions and the scaling are shown in figure $11(d)$. For this reason, case 3 is also considered with two beamlines delivering $33 \mathrm{MW}$ of $\mathrm{H}-\mathrm{NNBI}$. This case appears to access the H-mode (barely) using [12].

Both the full field DT and half-field bulk $\mathrm{H}$ cases use the ICRF frequency of $52.5 \mathrm{MHz}$, and assume the minority ion species is $\mathrm{He}^{3}$ at a density relative to the electron density of $2 \%$ for the DT and $3 \%$ for the pre-activation case 2 . The $\mathrm{He}^{3}$ absorption is at the fundamental frequency for the DT case and at the second harmonic for the half-field case. $\mathrm{He}^{3}$ heating is of interest for achieving a significant partition of the heating to thermal ion species, but it is considered optional for ITER. The ion partition increases with increasing $\mathrm{He}^{3}$ density, but high $\mathrm{He}^{3}$ density would dilute DT fuel. Also achieving large concentrations of $\mathrm{He}^{3}$ in the resonance layer may be very expensive for routine use. Another concern is that having a large partition of the heating to fast-ion species may be undesirable due to causing excessive losses and TAE drive.

The scalings for the $\mathrm{L} \rightarrow \mathrm{H}$ power threshold in $\mathrm{He}^{4}$ plasmas is controversial. The GLF23 predictions achieve H-mode with the ITPA scaling. Predicted temperature profiles are shown in figure $2(d)$. The minority ions also heat the electrons and thermal ions, and the accumulated heating is shown in figure 12 . Note that the minority heating can be negative at 


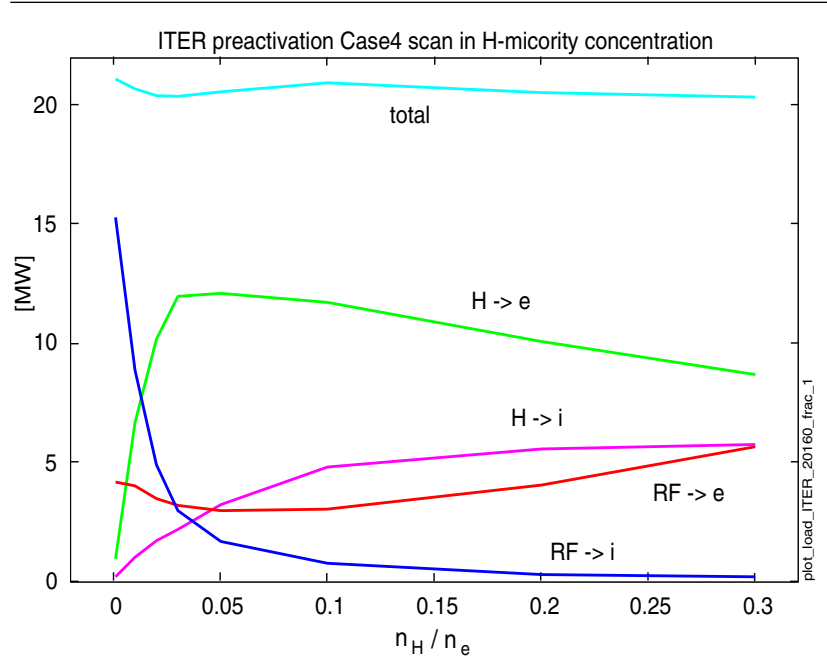

Figure 13. Total ICRF and minority heating powers for the pre-activation $\mathrm{He}^{4}$ case as a fraction of the $\mathrm{H}$ minority is varied. The summed ICRF and minority heating of thermal ions and electrons is needed for transport modelling. The total ICRF electron and ion heating powers are predicted to be relatively constant with fractions above $3 \%$.

large radii due to the plasma heating the minority instead of the usual vice-versa. Also the minority electron heating can be significantly larger than the ICRF electron heating, as in cases 3 and 4 . Only case 1 is predicted to have approximately equal total ion and electron heating. The importance of including the plasma heating by the minority ions is shown in figure 13 where the fraction of $\mathrm{H}$ minority in the bulk $\mathrm{He}^{4}$ case 4 is scanned. Although the direct RF-electron heating increases above 5\% as the $\mathrm{H}$ concentration increases, the $\mathrm{H}$ heating of electrons decreases.

A simplified antenna is used for the PTRANSP predictions. It is assumed to extend poloidally $1.83 \mathrm{~m}$, and is located $0.18 \mathrm{~m}$ outside the plasma boundary. For the heating results the toroidal spectrum at the antenna is approximated by two wavenumbers $n_{\phi}= \pm 27$, corresponding to $k_{\phi}$ is $4.229 \mathrm{~m}^{-1}$. The equivalent parallel index is 3.843. For the current-drive results one wavenumber $n_{\phi}=27$ is assumed. The ITER antenna design is composed of four columns of six short poloidal straps $(\simeq 0.27 \mathrm{~m})$. The top and bottom triplets of straps are driven in quadrature through an ELM-tolerant $3 \mathrm{~dB}$ hybrid splitter. In PTRANSP the vacuum vessel is assumed to be perfectly conducting, and thus the ICRF excites image currents in the vessel. These have small effects within the separatrix region if the antenna is not placed (numerically) too close to the vessel.

\section{Appendix B. Full-wave codes}

\section{B.1. AORSA}

The plasma state input files were pre-processed by a wrapper program that was developed for the Simulation of Wave Interactions with MHD (SWIM) project. For the energetic species $e_{\perp}$ and $e_{\|}$and an isotropic effective temperature given by equation (1) were used. Because of internal limitations for AORSA, only six species could be used. For the initial DT case 1 these are assumed to be electrons, T, D, $\mathrm{He}^{4}$ ash, $D_{\mathrm{nb}}$, alpha particles and $\mathrm{He}^{3}$ minority. Of these only electrons, $\mathrm{T}$ and $\mathrm{He}^{3}$ minority were significant. To assess heating of impurities the $D_{\text {nb }}$ and alpha particles were replaced with Ar and Be. The RF power in these species was small. Thus the plots only show electrons, $\mathrm{T}$ and $\mathrm{He}^{3}$ minority for clarity.

AORSA can be run with CQL3D, providing the possibility to model the minority distribution self-consistently. Previous ITER simulations with AORSA (e.g. $[15,16])$ used this mode. The ITER benchmarking cases were run both in this mode and using the effective isotropic temperature derived from the PTRANSP-FPPRF inputs. The CQL3D work can be done by interacting several times between AORAS and CQL3D. The results using FFPRF or CQL3D are very close.

This work uses two toroidal wavenumbers $\left(n_{\phi} \pm=27\right)$ to model the antenna current. Analysis using a full spectrum can also be done (cf the reference above). For these calculations several tens to 50 runs are required.

For the $2 \mathrm{D}$ version used for this study, the antenna is a current strap just inside the last closed flux surface, with a $\cos (k y \times y)$ profile for the current, where $k y=k 0 \times$ antlc, and antlc is a specified propagation constant, exactly as in TORIC. In a new version being developed the antenna can be outside the last closed flux surface. In 3D, with the full antenna spectrum, the antenna is some number of step function currents (4 for ITER, 12 for NSTX) with a given phasing between them. For the 2D version used for this study, the region between the antenna and plasma is not modelled separately, but is whatever the profiles give for that region up to $x=1$.

Results for the heating partitions are given in tables 69. Comparisons of the heating partitions with an alternative assumption of low $\mathrm{He}^{3}$ fraction are given in table 10 .

\section{B.2. CYRANO}

The CYRANO code [17] is very similar to TORIC. It uses finite elements in the radial direction and Fourier representation in the poloidal and toroidal dimensions. It only treats axisymmetric equilibrium so toroidal modes are independent, and solves the wave equation in the weak variational form ('Galerkin formalism'). The antenna model is idealized: infinitely thin straps in the radial direction, homogeneous antenna currents, includes radial feeders. The dielectric tensor includes corrections up to second order Larmor radius. It has been modified to include general (numeric) distribution functions to compute the dielectric response [18], and coupled to the quasi-linear Fokker-Planck code BATCH [32] to selfconsistently model the RF acceleration/quasi-linear diffusion problem. This has been successfully tested for fundamental $(N=1)$ heating of NBI ions in JET [18].

CYRANO has not been parallelized and runs on one (quad-core) CPU. The results here used 200 radial points $\times 128$ poloidal modes. Results for the heating partitions are given in tables 6-9. Comparisons of the heating partitions with an alternative assumption of low $\mathrm{He}^{3}$ fraction are given in table 10 .

\section{B.3. EVE}

The EVE code [19] is a full-wave solver based on a variational formulation of the Maxwell-Vlasov system. The wave particle interaction is described by a quasi-local plasma 
Table 12. EVE results for heating partitions (\%) for the pre-activation cases assuming isotropic or anisotropic effective temperatures. The bulk ion species is $\mathrm{H}$ for cases 2 and 3 and $\mathrm{He}^{4}$ for case 4.

\begin{tabular}{|c|c|c|c|c|c|c|}
\hline Case & $\begin{array}{l}\text { Effective } \\
T_{\min }\end{array}$ & $\begin{array}{l}\text { Bulk } \\
\text { ion } \\
\text { species }\end{array}$ & Electrons & Carbon & $\begin{array}{l}\text { Minority } \\
\text { species }\end{array}$ & $\begin{array}{l}\text { Beam } \\
\text { species }\end{array}$ \\
\hline \multirow[t]{2}{*}{2} & Anisotropic & 14.1 & 56.3 & 0.0 & 28.5 & 1.1 \\
\hline & Isotropic & 14.7 & 61.8 & 0.0 & 22.7 & 0.8 \\
\hline \multirow[t]{2}{*}{3} & anisotropic & 8.4 & 31.4 & 0.0 & 56.9 & 3.3 \\
\hline & Isotropic & 5.5 & 24.7 & 0.0 & 67.7 & 2.1 \\
\hline \multirow[t]{2}{*}{4} & Anisotropic & 1.0 & 25.0 & 0.1 & 73.9 & N.A. \\
\hline & Isotropic & 1.0 & 20.1 & 0.1 & 78.2 & N.A. \\
\hline
\end{tabular}

functional, in which a first order expanded version of the particle Hamiltonian has been implemented. The quadratic dependence of the functional on the interaction Hamiltonian makes EVE a second-order FLR code. The use of a Hamiltonian formulation and associated action-angle variables has the advantage of providing a common framework to the wave and the quasi-linear response calculation, making EVE the main element in a wave + kinetic package.

The core of the code is written in Fortran 90, parallelized, and runs on various clusters and supercomputers. It also features a post-processor written in Python. It is based on a toroidal geometry and can read analytical, HELENA, EFIT or ITM equilibria. The plasma profiles may be specified analytically, point-wise or read from the ITM data tree. The four cases are simulated with the effective temperatures taken as either isotropic equation (1) or anisotropic equation (2). Also the four cases were simulated assuming that the minority temperature equals the thermal ion temperature, i.e. that the plasma does not have an ICRF-heated tail.

Release version 1.4.7 is used for the results here. The grid used 460 radial and 512 poloidal points and 129 poloidal modes. The antenna is located between 8.38 and $8.39 \mathrm{~m}$, with feeder locations specified. The provided EFIT equilibrium and PTRANSP profiles have been directly used. Results for the heating partitions are given in tables 6-10. Comparisons of the alternative assumptions of isotropic or anisotropicMaxwellians for the minority temperatures are shown in table 12 .

\section{B.4. PSTELION}

PSTELION is a newly developed stellarator ICRF 3D full wave code $[20,33]$. The code solves wave excitation, propagation and absorption in 3D stellarator equilibrium high beta plasma in ion-cyclotron frequency range and lower. The MaxwellVlasov boundary value problem at the ICRF frequency range is solved on realistic equilibria in an elongated toroidal plasma geometry (produced by equilibrium solver as, for example, the VMEC code [34]).

The code solves the 3D Maxwell-Vlasov antenna-plasma conducting shell boundary value problem in non-orthogonal flux coordinates $(\Psi, \Theta, \phi)$ with $\Psi$ being the magnetic flux function, $\Theta$ and $\phi$ being the poloidal and toroidal angles, respectively. Basic physics such as wave refraction, reflection, diffraction and MC are self-consistently included, along with the fundamental and second harmonic ion and ion minority cyclotron resonances, two ion hybrid, Alfvén resonances, ELD and TTMP absorption. This is accomplished in a real confining magnetic field in a plasma major radius direction, in the toroidal and poloidal directions, through making use of ion and electron FLR effects in wave plasma response secondorder differential operators. In the Reduced Order Algorithm code option for the hot plasma, the dielectric kinetic tensor is used (FLR effects are accounted through reduced order scheme).

The numerical methods use an expansion of the solution in Fourier series over $\phi$ and $\Theta$ angles and solve the resulting ordinary differential equations in a radial-like $\Psi$ coordinate by a finite-difference method. The constructed discretization scheme is divergent free. The Fourier expansion over angle coordinates allows a construction of the parallel wavenumber $k_{\|}$and thereby describe the ICRF waves absorption by a hot plasma. The toroidal harmonics are tightly coupled with each other due to magnetic field inhomogeneity of stellarators in the toroidal direction. The code is developed in a manner that includes tokamaks and mirrors as the particular cases through a general metric tensor (provided by a plasma equilibrium solver) treatment of the wave equations. The resulting system of linear equations is solved by making use the ScaLAPACK library of parallelized linear algebra routines and direct use of the MPI interface.

PSTELION is coupled with the 2D STIION FokkerPlanck code [33] which uses flux-surface averaged RF absorbed specific power in a quasi-linear diffusion operator to calculate the minority ion distribution functions on chosen magnetic surfaces. Calculations requiring a large number of Fourier harmonics generate very large matrices. While these can be written to disk (for some particular sparse matrix solvers) when necessary and subsequently retrieved as required, this increases the computer time substantially.

PSTELION was built to use an up/down symmetric equilibrium. It was recently generalized to approximate up/down asymmetric equilibria using the VMEC2000 solver. Results for the heating partitions from the up/down asymmetric case 1 are given in table 6 . A comparison of results with alternative assumptions up/down symmetric and asymmetric magnetic flux geometry shows little effects in the heating partitions.

\section{B.5. TASK/WM}

TASK/WM [21] solves Maxwell's equations as a boundary value problem with magnetic flux coordinates in 3D configuration. Fourier mode expansion in the poloidal and toroidal directions, and finite-element method in the radial direction are used. Various kinds of dielectric tensors for any number of particle species can be used. The kinetic dielectric tensor includes the plasma dispersion function. FLR effects are included as a FW approximation. TASK/WF is coupled with the Fokker-Planck code TASK/FP and the orbit code GNET. The dielectric tensor for arbitrary velocity distribution function is treated in TASK/DP. Results for the heating partitions for the DT case 1 are given in table 6.

Future work planned is as follows: (1) arbitrary antenna configuration, (2) FLR effects in a integral form, (3) coupled with Fokker-Planck code, (4) parallel processing with TASK/WM/FP/DP. 
Table 13. TORIC results for heating partitions $(\%)$ for the pre-activation bulk $\mathrm{H}$ cases with two assumptions about the minority energy.

\begin{tabular}{lllll}
\hline $\begin{array}{l}\mathrm{He}^{3} \text { effective } \\
\text { temp }\end{array}$ & Hydrogen & Minority & Electrons & H-beams \\
\hline $10(\mathrm{keV})$ & 20.5 & 6 & 73 & 0.5 \\
$14(\mathrm{keV})$ & 19 & 36 & 49 & $\ll 1$ \\
\hline
\end{tabular}

\section{B.6. TORIC}

TORIC [22,23] solves Maxwell's equations in axisymmetric toroidal plasmas, assuming a constitutive relation (linear relation between high-frequency field and high-frequency plasma current) obtained from the linearized Vlasov equation by expanding the wave field in toroidal and poloidal Fourier components. The model includes propagation and damping of externally launched FWs, as well as of IBWs and ICWs excited by linear mode conversion (LMC) near ion-ion resonances. The absorption channels are fundamental and first-harmonic IC heating of ions, and ELD and transit time damping of electrons. Optionally, damping of the FW at higher IC harmonics can be simulated [35]. The coefficients of the wave equations are evaluated for arbitrary distribution functions using the information transmitted from the Fokker-Planck package SSFPQL [36]. Recent applications of the TORIC code can be found in [37].

The version 6 used for the benchmarking uses a new algorithm (originally invented for the numerical solution of the wave equations in the lower hybrid frequency range) implemented in the vacuum region surrounding the plasma. It solves Maxwell's equations exactly and with the same cubic finite elements as in the plasma, yet completely avoiding numerical pollution. As a result, the global power balance (agreement between the $J \times E$ loading of the antenna, the Poynting flux through the plasma surface, and the total power deposited in the plasma) is often appreciably more accurate than in previous TORIC versions. Thus in results for this study this agreement, which is a reliable indicator of convergence, was better than $0.3 \%$, in spite of the fact that, in order to execute on a laptop, only 128 points were used in the poloidal mesh, and thus only 63 poloidal Fourier modes in the representation of the fields. A further indication of convergence was the fact that the results did not significantly differ from those obtained with only 64 points and 31 Fourier modes, whose power balance was only marginally less accurate.

Results for the heating partitions for the DT case 1 are given in table 6. A comparison of results with alternative assumptions for the number of poloidal modes and radial grid is given in table 11. Results for pre-activation cases 2 and 3 exhibit a strong sensitivity to the $\mathrm{He}^{3}$ energy. The results with elevated $\mathrm{He}^{3}$ energy are the same as from PTRANSP.

TORIC was also used to study effects of variations in the minority ion effective temperatures. A strong sensitivity of the heating partitions is seen, shown in table 13 .

\section{References}

[1] Gormezano C. et al 2007 Nucl. Fusion 47 S285

[2] Houlberg W.A. et al 2005 Nucl. Fusion 451309
[3] Oikawa T. et al 2008 Proc. 22nd Int. Conf. on Fusion Energy, 2008 (Geneva, Switzerland 2008) (Vienna: IAEA) CD-ROM file IT/P6-5 and http://www-naweb.iaea.org/napc/ physics/FEC/FEC2008/html/index.htm

[4] Prater R. et al 2008 Nucl. Fusion 48035006

[5] Budny R.V. et al 2008 Nucl. Fusion 48075005

[6] Halpern F.D. et al 2008 Phys. Plasmas 15062505

[7] Budny R.V. 2009 Nucl. Fusion 49085008

[8] Budny R.V. 2012 Nucl. Fusion 52013001

[9] Lerche E. et al 2010 Experimental investigation of ICRF heating scenarios for ITER's half-field hydrogen phase performed in JET 37th EPS Conf. on Controlled Fusion and Plasma Physics (Dublin, Ireland June 2010) O4.121 http://ocs.ciemat.es/EPS2010PAP/pdf/O4.121.pdf

[10] Dumont R.J. (CEA, IRFM) 2010 private communication

[11] Ryter F. et al 2009 Nucl. Fusion 49062003

[12] Martin Y.R. et al 2008 J. Phys.: Conf. Ser. 123012033

[13] McCune D. NTCC Plasma State Module http://w3.pppl.gov/ntcc/PlasmaState/

[14] Lao L.U. 1990 Nucl. Fusion 301035

[15] Jaeger E.F., Berry L.A. and Harvey R.H. 2008 Phys. Plasmas 15072513

[16] Jaeger E.F. et al 2009 ICRF heating scenarios for the reduced magnetic field, non-nuclear phase of ITER Bulletin of the American Physical Society 51st Annual Meeting of the Division of Plasma Physics (Atlanta, GA, 2009)

[17] Lamalle P. 1994 PhD Thesis Université de Mons (1994) LPP-ERM/KMS Laboratory Report 101

Lamalle P.U. 1997 Plasma Phys. Control. Fusion 391409

[18] Lerche E. et al 2009 Plasma Phys. Control Fusion 51044006

[19] Dumont R.J. 2009 Nucl. Fusion 49075033

[20] Vdovin V.L. 2001 3D full wave code modelling of icrf plasma heating in large stellarators 28th European Conf. on Controlled Fusion and Plasma Physics (Madeira, Portugal, 18-22 June 2001) p4.058 (ECA) vol 25A pp 1541-4 http://www.cfn.ist.utl.pt/EPS2001/fin/ sessions/nav/Poster21 fr.html

[21] Batchelor D.A. et al 2007 Plasma Sci. Technol. 9312

[22] Brambilla M. 1999 Plasma Phys. Control. Fusion 411

[23] Wright J.C. et al 2004 Phys. of Plasmas 112473

[24] Hammett G. 1986 Fast ion studies of ion cyclotron heating in the PLT tokamak PhD Dissertation (Princeton, 1986), University Microfilms International No GAX86-12694.

[25] Jaeger E.F., Harvey R.H. and Berry L.A. 2006 Phys. Plasmas 13056101

[26] Harvey R.W. and McCoy M.G. 1998 IAEA Conf. Proc., Technical Committee Meeting on Advances in Simulation and Modeling of Thermonuclear Plasmas (Montreal, Canada, 15-18 June 1998) (Vienna, Austria: International Atomic Energy Agency) p 305

[27] Stix T. 1975 Nucl. Fusion 15737

[28] Hosea J. et al 1979 Phys. Rev. Lett. 431802

[29] Ehst D.A. and Karney C.F.F. 1991 Nucl. Fusion 311933

[30] Waltz R.E. et al 1997 Phys. Plasmas 42482

[31] Onjun T., Bateman G., Kritz A.H. and Hammett G. 2002 Phys. Plasmas 95018

[32] Van Eester D. 2001 J. Plasma Phys. 65407

[33] Vdovin V. 1980 The distribution function of resonant ions at ion cyclotron heating of plasma in tokamaks Proc. 2nd Joint Grenoble -Varenna Int. Symp. on Heating in Toroidal Plasmas (Como, Italy, September 1980) v 1 p 555

[34] Hirshman S.P. and Whitson J.C. 1983 Phys. Fluids 263553

[35] Brambilla M 2002 Plasma Phys. Control. Fusion 442423

[36] Brambilla M. and Bilato R. 2009 Nucl. Fusion 49085004

[37] Wright J.C. et al 2005 Nucl. Fusion 451411 